\title{
AN ANALYSIS OF THE EMBEDDED DISCONTINUOUS GALERKIN METHOD FOR SECOND ORDER ELLIPTIC PROBLEMS
}

\author{
BERNARDO COCKBURN *, JOHNNY GUZMÁN †, SEE-CHEW SOON ${ }^{\ddagger}$, AND HENRYK \\ K. STOLARSKI §
}

\begin{abstract}
The embedded discontinuous Galerkin methods are obtained from hybridizable discontinuous Galerkin methods by a simple change of the space of the hybrid unknown. In this paper, we consider embedded methods for second-order elliptic problems obtained from hybridizable discontinuous methods by changing the space of the hybrid unknown from discontinuous to continuous functions. This change results in a significantly smaller stiffness matrix whose size and sparsity structure coincides with those of the stiffness matrix of the statically condensed continuous Galerkin method. It is shown that this computational advantage has to be balanced against the fact that the approximate solutions for the scalar variable and its flux lose each a full order of convergence. Indeed, we prove that, if polynomials of degree $k \geq 1$ are used for the original hybridizable discontinuous Galerkin method, its approximations to the scalar variable and its flux converge with order $k+2$ and $k+1$, respectively, whereas those of the corresponding embedded discontinuous Galerkin method converge with orders $k+1$ and $k$, respectively, only. We also provide numerical results comparing the relative efficiency of the methods.
\end{abstract}

Key words. finite element methods, mixed methods, discontinuous Galerkin methods, Lagrange multipliers

AMS subject classifications. 65N30, 65M60, 35L65

1. Introduction. In this paper, we continue the study of the embedded discontinuous Galerkin (EDG) methods started in [11] in the framework of linear shells and carry out an a priori error analysis in the framework of the model second-order elliptic problem

$$
\begin{aligned}
\boldsymbol{q}+\nabla u=0 & & \text { in } \Omega, \\
\nabla \cdot \boldsymbol{q}=f & & \text { in } \Omega, \\
u=g & & \text { on } \partial \Omega_{D}, \\
\boldsymbol{q} \cdot \boldsymbol{n}=\mathbf{q}_{\mathrm{N}} & & \text { on } \partial \Omega_{N} .
\end{aligned}
$$

Here $\Omega \subset \mathbb{R}^{d}$ is a polyhedral domain $(d \geq 2), f \in L^{2}(\Omega)$. As usual, the $L^{2}$ estimates for the error in the approximation of $u$ require elliptic regularity results that hold when $\Omega$ is convex and $\partial \Omega_{D}=\partial \Omega$.

The EDG methods are obtained from hybridizable discontinuous Galerkin by simply reducing the space of the hybrid unknown; see [11] and [9]. Since the only degrees of freedom that are globally coupled are precisely those of this unknown, this reduction renders the computational complexity of the EDG method smaller than that of the HDG method it is obtained from. In this paper, we show that the price we

*School of Mathematics, University of Minnesota, Minneapolis, MN 55455, USA, email: cockburn@math.umn.edu. Supported in part by the National Science Foundation (Grant DMS0712955) and by the University of Minnesota Supercomputing Institute.

${ }^{\dagger}$ School of Mathematics, University of Minnesota, Minneapolis, MN 55455, USA, email: guzma033@umn.edu. Supported by an NSF Mathematical Science Postdoctoral Research Fellowship (DMS-0503050).

${ }^{\ddagger}$ Department of Civil Engineering, University of Minnesota, Minneapolis, MN 55455, USA, email: soon0005@umn.edu.

$\S$ Department of Civil Engineering, University of Minnesota, Minneapolis, MN 55455, USA, email: stola001@tc.umn.edu. 
have to pay for this computational advantage is the loss of a full power in the order of convergence of the approximations of both the potential and its flux. Note that we prove these results for any space dimension $d \geq 2$.

To better describe our results, let us briefly review the recent developments of DG methods for second-order elliptic equations. All the methods considered in the unifying analysis of DG methods for second-order elliptic problems in [1], which use polynomial approximations of degree $k$ for both the potential and the flux, converge with the optimal order of $k+1$ for the potential and with the suboptimal order of $k$ for the flux. Since the classic continuous Galerkin method needs considerably less degrees of freedom, on the same mesh, and converges with exactly the same orders, the use of DG methods for second-order elliptic equations has been judged as not being particularly advantageous; see, for example, [15]. In [9], the HDG methods were introduced to address this criticism.

The HDG methods. These methods are devised in such a way that the globally coupled degrees of freedom are only those of the so-called numerical trace of the potential. To show how this is achieved, we need to introduce some notation.

We denote by $\Omega_{h}=\{K\}$ a triangulation of the domain $\Omega$ of shape-regular simplexes $K$ and set $\partial \Omega_{h}:=\left\{\partial K: K \in \Omega_{h}\right\}$. We associate to this triangulation the set of interior faces $\mathscr{E}_{h}^{i}$ and the set of boundary faces $\mathscr{E}_{h}$. We say that $e \in \mathscr{E}_{h} i$ if there are two simplexes $K^{+}$and $K^{-}$in $\Omega_{h}$ such that $e=\partial K^{+} \cap \partial K^{-}$, and we say that $e \in \mathscr{E}_{h} \partial$ if there is a simplex in $\Omega_{h}$ such that $e=\partial K \cap \partial \Omega$. We set $\mathscr{E}_{h}:=\mathscr{E}_{h} i \cup \mathscr{E}_{h} \partial$. We are also going to use the following notation

$$
\begin{aligned}
(\zeta, \omega)_{\Omega_{h}} & :=\sum_{K \in \Omega_{h}} \int_{K} \zeta(x) \omega(x) d x, & & \forall \zeta, \omega \in H^{1}\left(\Omega_{h}\right), \\
(\boldsymbol{\sigma}, \boldsymbol{v})_{\Omega_{h}} & :=\sum_{i=1}^{d}\left(\sigma_{i}, v_{i}\right)_{\Omega_{h}} & & \forall \boldsymbol{\sigma}, \boldsymbol{v} \in \boldsymbol{H}^{1}\left(\Omega_{h}\right), \\
\langle\boldsymbol{v} \cdot \boldsymbol{n}, \mu\rangle_{\partial \Omega_{h}}: & : \sum_{K \in \Omega_{h}} \int_{\partial K} \boldsymbol{v}(\gamma) \cdot \boldsymbol{n} \mu(\gamma) d \gamma & & \forall(\boldsymbol{v}, \mu) \in \boldsymbol{L}^{2}\left(\partial \Omega_{h}\right) \times L^{2}\left(\partial \Omega_{h}\right) .
\end{aligned}
$$

We are now ready to introduce the HDG methods we are interested in.

The HDG methods seek an approximation to $\left(\boldsymbol{q}, u,\left.u\right|_{\mathscr{E}_{h}}\right),\left(\boldsymbol{q}_{h}, u_{h}, \widehat{u}_{h}\right)$ in the space $\boldsymbol{V}_{h} \times W_{h} \times \mathrm{M}_{h}$ where

$$
\begin{array}{llll}
\boldsymbol{V}_{h}=\left\{\boldsymbol{v} \in \boldsymbol{L}^{2}(\Omega):\right. & \left.\boldsymbol{v}\right|_{K} \in \mathcal{P}^{k}(K) & \forall & \left.K \in \Omega_{h}\right\}, \\
W_{h}=\left\{w \in L^{2}(\Omega):\right. & \left.w\right|_{K} \in \mathcal{P}^{k}(K) & \forall & \left.K \in \Omega_{h}\right\}, \\
\mathrm{M}_{h}=\left\{\mu \in L^{2}\left(\mathscr{E}_{h}\right):\right. & \left.\mu\right|_{e} \in \mathcal{P}^{k}(e) & \forall & \left.e \in \mathscr{E}_{h}\right\} .
\end{array}
$$

Here $\mathcal{P}^{k}(S)$ denotes the set of polynomials of degree $k$ on $S$ and $\mathcal{P}^{k}(S)=\left[\mathcal{P}^{k}(S)\right]^{d}$. The approximation $\left(\boldsymbol{q}_{h}, u_{h}, \widehat{u}_{h}\right)$ is determined by requiring that

$$
\begin{aligned}
\left(\boldsymbol{q}_{h}, \boldsymbol{v}\right)_{\Omega_{h}}-\left(u_{h}, \nabla \cdot \boldsymbol{v}\right)_{\Omega_{h}}+\left\langle\widehat{u}_{h}, \boldsymbol{v} \cdot \boldsymbol{n}\right\rangle_{\partial \Omega_{h}} & =0, \\
-\left(\boldsymbol{q}_{h}, \nabla \omega\right)_{\Omega_{h}}+\left\langle\widehat{\boldsymbol{q}}_{h} \cdot \boldsymbol{n}, \omega\right\rangle_{\partial \Omega_{h}} & =(f, \omega)_{\Omega_{h}}, \\
\left\langle\widehat{\boldsymbol{q}}_{h} \cdot \boldsymbol{n}, \mu\right\rangle_{\partial \Omega_{h}} & =\left\langle\mathbf{q}_{\mathrm{N}}, \mu\right\rangle_{\partial \Omega_{N}},
\end{aligned}
$$

for all $(\boldsymbol{v}, \omega, \mu) \in \boldsymbol{V}_{h} \times W_{h} \times M_{h}$, where

$$
\begin{array}{ll}
\widehat{\boldsymbol{q}}_{h}:=\boldsymbol{q}_{h}+\tau\left(u_{h}-\widehat{u}_{h}\right) \boldsymbol{n} & \text { on } \partial \Omega_{h}, \\
\widehat{u}_{h}:=\mathrm{P}_{\partial} g & \text { on } \partial \Omega_{D},
\end{array}
$$


where, for each face $e \in \mathscr{E}_{h} \cap \partial \Omega_{D},\left.\mathrm{P}_{\partial}\right|_{e}$ is the $L^{2}$-projection into $\mathcal{P}^{k}(e)$. Here, $\tau$ is a piecewise-constant function on $\partial \Omega_{h}$; it is thus a double-valued function on interior faces.

Note that, by using the first two equations defining the method, (1.3a) and (1.3b), we can express $\left(\boldsymbol{q}_{h}, u_{h}\right)$ in terms of $\widehat{u}_{h}$ and $f$ in an element-by-element fashion; we have to use the definition of the numerical trace of the flux (1.3d), of course. Then, the equation (1.3c) can be rewritten in terms $\widehat{u}_{h}$ and $f$ only. In [9] such an equation was shown to be written in variational form as follows. First, define $I_{h} g$ on $\mathscr{E}_{h}$ to be the extension by zero of $\mathrm{P}_{\partial} g$, and set $\widehat{u}_{h}=\lambda_{h}+I_{h} g$. Then $\lambda_{h}$ is the function in $M_{h}$, where

$$
M_{h}=\left\{\mu \in \mathrm{M}_{h}:\left.\quad \mu\right|_{\partial \Omega_{D}}=0\right\}
$$

such that

$$
a_{h}\left(\lambda_{h}, \mu\right)=b_{h}\left(I_{h} g, f ; \mu\right) \quad \forall \quad \mu \in M_{h}
$$

In this way, both $\boldsymbol{q}_{h}$ and $u_{h}$ are eliminated from the equations and a single variational formulation is obtained for the unknown $\lambda_{h}$. This renders these HDG methods as efficiently implementable as the hybridized versions of the Raviart-Thomas (RT) [14], see also [8], and the Brezzi-Douglas-Marini (BDM) [2] mixed methods of corresponding degree.

The EDG methods. In an effort to further reduce the computational complexity of these methods, the EDG methods were introduced. Indeed, the EDG methods are obtained from the HDG methods by simply reducing the space of the numerical trace $\widehat{u}_{h}, \mathrm{M}_{h}$. The first EDG method was introduced in [11] in the framework of linear shell theory by forcing the space of numerical traces of the displacement to be continuous. The resulting method was shown to retain the ability of the local discontinuous Galerkin (LDG) method for shells introduced in [12] to bypass locking, while being more efficiently implemented. Indeed, its stiffness matrix is of the size and structure than that of the statically condensed continuous Galerkin method. Thus, this EDG method could be easily incorporated in existing commercial codes with no essential difficulty.

Let us show how the EDG methods we consider here are obtained. First, we reduce the space $\mathrm{M}_{h}$ to the space of functions in $\mathrm{M}_{h}$ which are also continuous in $\mathscr{E}_{h}$, namely, to

$$
\widetilde{\mathrm{M}}_{h}=\mathrm{M}_{h} \cap \mathcal{C}^{0}\left(\mathscr{E}_{h}\right)
$$

We then take $\widehat{u}_{h}=\widetilde{\lambda}_{h}+\widetilde{I}_{h} g$, where $\widetilde{I}_{h} g$ is an approximation in $\widetilde{M}_{h}$ of (the extension to $\mathscr{E}_{h}$ by zero of) $g$, and take $\widetilde{\lambda}_{h}$ as the element of $\widetilde{M}_{h}$, where

$$
\widetilde{M}_{h}=\left\{\mu \in \widetilde{M}_{h}:\left.\quad \mu\right|_{\partial \Omega_{D}}=0\right\}
$$

satisfying

$$
a_{h}\left(\mathcal{J}_{h} \widetilde{\lambda}_{h}, \mathcal{\partial}_{h} \widetilde{\mu}\right)=b_{h}\left(\mathcal{J}_{h} \widetilde{I}_{h} g, f ; \partial_{h} \widetilde{\mu}\right) \quad \forall \quad \widetilde{\mu} \in \widetilde{M}_{h},
$$

where $\mathcal{J}_{h}: \widetilde{M}_{h} \rightarrow M_{h}$ is the identity operator representing the natural injection from $\widetilde{M}_{h}$ into $M_{h}$. Then we replace $\lambda_{h}$ by $\widetilde{\lambda}_{h}$ in the equations (1.3a), (1.3b) and solve for $\left(\boldsymbol{q}_{h}, u_{h}\right)$, by using $(1.3 \mathrm{c})$, in an element-by-element fashion. We can thus see that 
solving for $\widetilde{\lambda}_{h}$ is less computationally expensive than solving for $\lambda_{h}$ since $\widetilde{M}_{h}$ is a smaller space than $M_{h}$. This suggests the possibility that EDG methods could be superior to the HDG methods they are deduced from.

The enhanced accuracy of the HDG methods. Parallel to the abovementioned development, HDG methods were discovered to be not only more efficiently implementable than all the previously known DG methods for second-order elliptic problems, but also more accurate. The first of those methods in several space dimensions was the so-called single face-hybridizable (SFH) discontinuous Galerkin method; see [7]. It lies in between the Raviart-Thomas (RT) [14] and the Brezzi-Douglas-Marini (BDM) [2] mixed methods of corresponding degree, and shares with them several of their remarkable convergence properties. It was proven to converge with the optimal order of $k+1$ for the flux, and to provide a new approximation for the potential, $u_{h}^{\star}$, converging with order $k+2$ for any $k \geq 1$. Soon after, in [10], a wider class of DG methods having the above-mentioned properties was found which included non-LDG methods previously studied in [4] as well as the HDG methods considered here (1.3). It was proven therein that the local stabilization parameter $\tau$ can be chosen in such a way that, if polynomials of degree $k$ are used for both the potential and the flux, we can obtain approximations that converge with order $k+2$ and $k+1$ to the potential and the flux, respectively, for $k \geq 1$. In this paper, we prove that the corresponding EDG method, provides approximations converging only with orders $k+1$ and $k$, respectively. We also argue that this takes places because, unlike the HDG methods, the numerical trace $\widehat{\boldsymbol{q}}_{h}$ for the EDG methods is not single valued.

We also show that, if the stabilization parameter $\tau$ is chosen to be big enough, the HDG as well as the corresponding EDG method provide approximations for the potential and flux converging only with orders $k+1$ and $k$. A similar result was reported for the so-called multiscale discontinuous Galerkin (MDG) method introduced in [13] in the framework of convection-diffusion equations, and analyzed later in [3]. Indeed, since these methods were introduced by using DG methods providing approximations for the potential and flux converging with orders $k+1$ and $k$, no significant difference was observed between the approximations provided donor DG method and those of the resulting MDG method; see [13].

The organization of the paper is as follows. In Section 2, we rewrite our EDG methods in a way amenable to analysis and present and briefly discuss our main theoretical results. In Sections 3 and 4, we provide detailed proofs of the estimates of the error in the flux and in the potential, respectively. In Section 5, we provide numerical results showing that our results are sharp, and end with some concluding remarks in Section 5.

\section{The main results.}

A rewriting of the EDG methods. It is not difficult to see that the approximation to $\left(\boldsymbol{q}, u,\left.u\right|_{\mathscr{E}_{h}}\right),\left(\boldsymbol{q}_{h}, u_{h}, \widehat{u}_{h}\right)$, given the EDG methods described in the introduction can be characterized as the element of the space $\boldsymbol{V}_{h} \times W_{h} \times \widetilde{\mathrm{M}}_{h}$ such that

$$
\begin{aligned}
\left(\boldsymbol{q}_{h}, \boldsymbol{v}\right)_{\Omega_{h}}-\left(u_{h}, \nabla \cdot \boldsymbol{v}\right)_{\Omega_{h}}+\left\langle\widehat{u}_{h}, \boldsymbol{v} \cdot \boldsymbol{n}\right\rangle_{\partial \Omega_{h}} & =0 \\
-\left(\boldsymbol{q}_{h}, \nabla \omega\right)_{\Omega_{h}}+\left\langle\widehat{\boldsymbol{q}}_{h} \cdot \boldsymbol{n}, \omega\right\rangle_{\partial \Omega_{h}} & =(f, \omega)_{\Omega_{h}}, \\
\left\langle\widehat{\boldsymbol{q}}_{h} \cdot \boldsymbol{n}, \mu\right\rangle_{\partial \Omega_{h}} & =\left\langle\mathbf{q}_{\mathrm{N}}, \mu\right\rangle_{\partial \Omega_{N}},
\end{aligned}
$$


for all $(\boldsymbol{v}, \omega, \mu) \in \boldsymbol{V}_{h} \times W_{h} \times \widetilde{M}_{h}$, where

$$
\begin{array}{ll}
\widehat{\boldsymbol{q}}_{h}:=\boldsymbol{q}_{h}+\tau\left(u_{h}-\widehat{u}_{h}\right) \boldsymbol{n} & \text { on } \partial \Omega_{h}, \\
\widehat{u}_{h}:=\widetilde{I}_{h} g & \text { on } \partial \Omega_{D} .
\end{array}
$$

A priori error estimates for the flux $\boldsymbol{q}$. We begin with an a priori estimate of the error in the approximation of the flux $\boldsymbol{q}$. To state it, we need some notation. We set

$$
\tau_{K}:=\left.\max \tau\right|_{\partial K},
$$

and denote by $e_{K}^{\tau}$ the face of $K$ on which $\left.\tau\right|_{\partial K}$ attains its maximum. As shown in [9], for the EDG method to be well-defined we require that $\tau_{K}>0$ for every $K \in \Omega_{h}$. Finally, we set

$$
\begin{aligned}
& \kappa=\max _{K \in \Omega_{h}}\left(\frac{h_{K}}{\tau_{K}}\right)^{1 / 2}, \\
& \rho=\max _{K \in \Omega_{h}}\left(h_{K} \bar{\tau}_{K}\right)^{1 / 2},
\end{aligned}
$$

where $\bar{\tau}_{K}$ denotes the maximum of $\tau$ on $\partial K \backslash e_{K}^{\tau}$. We are now ready to state our first result.

TheOREM 2.1. Let $\boldsymbol{q}_{h}$ be the EDG approximation to $\boldsymbol{q}$. Then

$$
\left\|\boldsymbol{q}_{h}-\boldsymbol{q}\right\|_{\boldsymbol{L}^{2}\left(\Omega_{h}\right)} \leq C_{1} h^{k}
$$

where

$$
C_{1}=C(\kappa+h)|\boldsymbol{q}|_{H^{k+1}\left(\Omega_{h}\right)}+C|u|_{H^{k+1}\left(\Omega_{h}\right)} .
$$

We also have

$$
\left\|\boldsymbol{q}_{h}-\boldsymbol{q}\right\|_{\boldsymbol{L}^{2}\left(\Omega_{h}\right)} \leq C_{2} h^{k},
$$

where

$$
C_{2}=C((1+\rho) h+\rho \kappa)|\boldsymbol{q}|_{\boldsymbol{H}^{k+1}\left(\Omega_{h}\right)}+C(1+\rho)|u|_{H^{k+1}\left(\Omega_{h}\right)} .
$$

Note that the above result gives error estimates of size $O\left(h^{k}\right)$ for $\boldsymbol{q}$ for a variety of choices of the local stabilization parameter $\tau$. For example, it holds when

- The parameter $\tau$ is taken independently of the size of the elements as in the HDG methods considered in [10]. In this case, the estimate of the flux is given by the second inequality where $\kappa$ as well as $\rho$ are of order $h^{1 / 2}$. Again, we obtain a rate convergence of order $h^{k}$ for the EDG method which has to be contrasted with the order of $h^{k+1}$ of the corresponding HDG method.

- The parameter $\left.\tau\right|_{\partial K}$ is taken equal to zero on all the faces $e$ of $\partial K$ except on one arbitrarily chosen face $e_{K}^{\tau}$. The corresponding HDG method is the SFH method introduced in [7]. Note that in this case, $\rho=0$ and the estimate of the error in the flux is given by the second inequality. Note that it is independent of the value of $\tau$ on $e_{K}^{\tau}$ in full agreement with a similar result for the SFH method. On the other hand, let us recall that the SFH approximate flux converges with the optimal rate of order $h^{k+1}$. 
- The parameter $\left.\tau\right|_{\partial K}$ is taken to be proportional to $1 / h_{K}$. In this case, the first inequality with $\kappa=h$ gives a rate of convergence of order $h^{k}$. This is the same rate of convergence proven to hold for the original HDG method in [4].

- The parameter $\tau$ is let to go to infinity, case in which the EDG method becomes the classic continuous Galerkin method. In this case, the first inequality with $\kappa=0$ says that the rate of convergence is of order $h^{k}$, as expected.

In all of the above cases, the rate of convergence of the approximate flux is of order $h^{k}$, even in the case in which the approximate flux of the corresponding HDG method converges with order $h^{k+1}$.

A priori error estimates for $u$. Now we obtain a priori estimates for the approximation $\left(u_{h}, \widehat{u}_{h}\right)$ to $\left(\left.u\right|_{\Omega},\left.u\right|_{\mathscr{E}_{h}}\right)$. As usual, we assume that the dual problem satisfies the following $H^{2}$-regularity result: If $(\psi, \sigma)$ solves the following dual problem

$$
\begin{aligned}
\boldsymbol{\psi}+\nabla \sigma=0 & \text { in } \Omega, \\
\nabla \cdot \boldsymbol{\psi}=\theta & \text { in } \Omega, \\
\sigma=0 & \text { on } \partial \Omega_{D} \\
\boldsymbol{\psi} \cdot \boldsymbol{n}=0 & \text { on } \partial \Omega_{N}
\end{aligned}
$$

then

$$
\|\boldsymbol{\psi}\|_{\boldsymbol{H}^{1}\left(\Omega_{h}\right)}+\|\sigma\|_{H^{2}\left(\Omega_{h}\right)} \leq C\|\theta\|_{L^{2}\left(\Omega_{h}\right)} .
$$

First we give error estimates for $u-u_{h}$ in terms of the error in $\boldsymbol{q}-\boldsymbol{q}_{h}$.

THEOREM 2.2. Let $u_{h}$ be approximation to $u$ given by the EDG method. If the regularity result (2.5) holds, then

$$
\begin{aligned}
\left\|u-u_{h}\right\|_{L^{2}\left(\Omega_{h}\right)} \leq & C h\left\|\boldsymbol{q}-\boldsymbol{q}_{h}\right\|_{\boldsymbol{L}^{2}\left(\Omega_{h}\right)}+\left\|g-\tilde{I}_{h} g\right\|_{L^{2}\left(\partial \Omega_{D}\right)} \\
& +C \kappa\left(h^{k+1}+\kappa h^{k}\right)|\boldsymbol{q}|_{\boldsymbol{H}^{k+1}\left(\Omega_{h}\right)}+C\left(\kappa h^{k}+h^{k+1}\right)\|u\|_{H^{k+1}\left(\Omega_{h}\right)} .
\end{aligned}
$$

As the above result suggests, $\left\|u-u_{h}\right\|_{L^{2}\left(\Omega_{h}\right)}$ depends strongly on the penalty parameter $\tau$. For example, if $\left.\tau\right|_{\partial K} \equiv h_{K}$ for all $K \in \Omega_{h}$, in which case we have $\kappa=1$, the $L^{2}$ error estimate is not optimal. However, if $\left.\tau\right|_{\partial K} \equiv \frac{1}{h_{K}}$, and hence $\kappa=h$, we recover optimal error estimates.

In the next theorem we present error estimates for the $L^{2}$-projection of $u-u_{h}$ into polynomials of degree $k-1$, which we denote by $\mathrm{P}^{k-1}\left(u-u_{h}\right)$. We will see that this quantity depends more weakly on the penalty parameter $\tau$. The next theorem also contains error estimates for the numerical trace $\widehat{u}_{h}$. To state it, we need to introduce the following quantity:

$$
\|\eta\|_{L^{2}\left(\partial \Omega_{h} ; h\right)}:=\left(\sum_{K \in \Omega_{h}} h_{K}\|\eta\|_{L^{2}(\partial K)}^{2}\right)^{1 / 2} .
$$

THEOREM 2.3. Let $\left(u_{h}, \widehat{u}_{h}\right)$ be approximation to $\left(\left.u\right|_{\Omega_{h}},\left.u\right|_{\mathscr{E}_{h}}\right)$ given by the EDG method. If the regularity result (2.5) holds, then

$$
\begin{aligned}
&\left\|\mathrm{P}^{k-1}\left(u-u_{h}\right)\right\|_{L^{2}\left(\Omega_{h}\right)} \leq C h\left\|\boldsymbol{q}-\boldsymbol{q}_{h}\right\|_{\boldsymbol{L}^{2}\left(\Omega_{h}\right)}+\left\|\mathrm{P}_{\partial} g-\tilde{I}_{h} g\right\|_{L^{2}\left(\partial \Omega_{D}\right)}, \\
&\left\|\mathrm{P}_{\partial} u-\widehat{u}_{h}\right\|_{L^{2}\left(\partial \Omega_{h} ; h\right)} \leq C h\left\|\boldsymbol{q}-\boldsymbol{q}_{h}\right\|_{\boldsymbol{L}^{2}\left(\Omega_{h}\right)}+\left\|\mathrm{P}_{\partial} g-\tilde{I}_{h} g\right\|_{L^{2}\left(\partial \Omega_{D}\right)} .
\end{aligned}
$$


We see that, unlike the HDG methods considered in [7] and [10], since the flux does not converge with optimal order, it is not possible to use an element-by-element postprocessing to obtain a new approximation $u_{h}^{\star}$ converging with order $h^{k+2}$. Thus, the price to pay for employing the EDG method is the loss of accuracy in such postprocessed approximation too.

Let us end by pointing out that when $k=1$, it is not difficult to see that the numerical trace $\widehat{u}_{h}$ coincides with the restriction to $\mathscr{E}_{h}$ of the continuous Galerkin approximation. This is not true in general for $k>1$. See the Appendix for a detailed proof of these facts.

\section{Proofs.}

3.1. Preliminaries: The error equations. Since the basic tool for our error analysis are the equations satisfied by the errors $\left(\boldsymbol{e}_{\boldsymbol{q}}, e_{u}\right):=\left(\boldsymbol{q}-\boldsymbol{q}_{h}, u-u_{h}\right)$, we write them here. From the characterization of the EDG method given by equations (2.1), we can easily see that they are the following:

$$
\begin{array}{r}
\left(\boldsymbol{e}_{\boldsymbol{q}}, \boldsymbol{v}\right)_{\Omega_{h}}-\left(e_{u}, \nabla \cdot \boldsymbol{v}\right)_{\Omega_{h}}+\left\langle\widehat{e}_{u}, \boldsymbol{v} \cdot \boldsymbol{n}\right\rangle_{\partial \Omega_{h}}=0, \\
-\left(\boldsymbol{e}_{\boldsymbol{q}}, \nabla \omega\right)_{\Omega_{h}}+\left\langle\widehat{\boldsymbol{e}}_{\boldsymbol{q}} \cdot \boldsymbol{n}, \omega\right\rangle_{\partial \Omega_{h}}=0, \\
\left\langle\widehat{\boldsymbol{e}}_{\boldsymbol{q}} \cdot \boldsymbol{n}, \mu\right\rangle_{\partial \Omega_{h}}=0,
\end{array}
$$

for all $(\boldsymbol{v}, \omega, \mu) \in \boldsymbol{V}_{h} \times W_{h} \times \widetilde{M}_{h}$, where

$$
\begin{array}{ll}
\widehat{\boldsymbol{e}}_{\boldsymbol{q}}:=\boldsymbol{e}_{\boldsymbol{q}}-\tau\left(u_{h}-\widehat{u}_{h}\right) \boldsymbol{n} & \text { on } \partial \Omega_{h}, \\
\widehat{e}_{u}:=u-\widehat{u}_{h} & \text { on } \mathscr{E}_{h} .
\end{array}
$$

3.2. Proof of the estimate of $\boldsymbol{q}-\boldsymbol{q}_{h}$, Theorem 2.1. To prove Theorem 2.1, we are going to proceed in several steps.

Step 1: A simple standard energy estimate. We start by using the standard energy argument to obtain a very simple estimate of the error in the flux.

In order to state the result we need to define the projection introduced in [5]; it is similar to the well-known Raviart-Thomas projection but has less constraints since its space is of a smaller dimension. Thus, for any $\boldsymbol{q} \in \boldsymbol{H}^{1}\left(\Omega_{h}\right)$, its projection $\boldsymbol{\Pi} \boldsymbol{q} \in \boldsymbol{V}_{h}$, is defined on each element $K \in \Omega_{h}$ by

$$
\begin{aligned}
(\boldsymbol{q}-\boldsymbol{\Pi} \boldsymbol{q}, \boldsymbol{v}) & =0, \quad \forall \quad \boldsymbol{v} \in \mathcal{P}^{k-1}(K), \\
\langle(\boldsymbol{q}-\boldsymbol{\Pi} \boldsymbol{q}) \cdot \boldsymbol{n}, \mu\rangle_{e} & =0, \quad \forall \quad \mu \in \mathcal{P}^{k}(e), \text { for all faces } e \text { on } \partial K \backslash e_{K}^{\tau} .
\end{aligned}
$$

We also denote by $\mathbf{P}_{\partial}$ the $L^{2}$-projection into $\mathrm{M}_{h}$.

LEMMA 3.1. Let $\left(\boldsymbol{q}_{h}, u_{h}, \widehat{u}_{h}\right)$ be the approximation given by the EDG method. Let $\Pi \boldsymbol{q}$ be the projection of $\boldsymbol{q}$ defined in (3.2). Moreover, let $I_{h} u \in \widetilde{\mathrm{M}}_{h}$ be a continuous interpolant of $u$ such that $I_{h} u=\widetilde{I}_{h} g$ on $\partial \Omega_{D}$. Then,

$$
\begin{aligned}
\left\|\boldsymbol{q}-\boldsymbol{q}_{h}\right\|_{\boldsymbol{L}^{2}\left(\Omega_{h}\right)}^{2}+ & \sum_{K \in \Omega_{h}}\left\|\tau^{1 / 2}\left(u_{h}-\widehat{u}_{h}\right)\right\|_{L^{2}(\partial K)}^{2} \\
& \leq C\|\boldsymbol{\Pi} \boldsymbol{q}-\boldsymbol{q}\|_{\boldsymbol{L}^{2}\left(\Omega_{h}\right)}^{2}+C\left\|\nabla\left(I_{h} u-u\right)\right\|_{\boldsymbol{L}^{2}\left(\Omega_{h}\right)}^{2} \\
& +C \sum_{K \in \Omega_{h}} \frac{1}{\tau_{K}}\left\|\left(\boldsymbol{\Pi} \boldsymbol{q}-\mathbf{P}_{\partial} \boldsymbol{q}\right) \cdot \boldsymbol{n}\right\|_{L^{2}\left(e_{K}^{\tau}\right)}^{2} .
\end{aligned}
$$


A direct consequence of these results and of the approximation results for $\boldsymbol{\Pi}$, obtained in [7], and those for the interpolation operator $I_{h}$, which are standard, is the following.

Corollary 3.2. Assume $\boldsymbol{q} \in \boldsymbol{H}^{k+1}\left(\Omega_{h}\right)$ and that $u \in H^{k+1}\left(\Omega_{h}\right)$, then

$$
\begin{aligned}
\left\|\boldsymbol{q}-\boldsymbol{q}_{h}\right\|_{\boldsymbol{L}^{2}\left(\Omega_{h}\right)} & +\left(\sum_{K \in \Omega_{h}}\left\|\tau^{1 / 2}\left(u_{h}-\widehat{u}_{h}\right)\right\|_{L^{2}(\partial K)}^{2}\right)^{1 / 2} \\
& \leq C\left(h^{k+1}+\kappa h^{k}\right)|\boldsymbol{q}|_{\boldsymbol{H}^{k+1}\left(\Omega_{h}\right)}+C h^{k}|u|_{H^{k+1}\left(\Omega_{h}\right)} .
\end{aligned}
$$

This implies that if $\kappa$ is uniformly bounded, that is, if there exists a $c \operatorname{such} \tau_{K} \geq c h_{K}$ for all $K \in \Omega_{h}$, then the error in $\boldsymbol{q}$ is of order $h^{k}$.

Proof. [Proof of Lemma 3.1] If in the first error equation (3.1a), we take $\boldsymbol{v}:=\boldsymbol{\Pi} \boldsymbol{e}_{\boldsymbol{q}}$, we obtain,

$$
\left(\boldsymbol{e}_{\boldsymbol{q}}, \boldsymbol{\Pi} \boldsymbol{e}_{\boldsymbol{q}}\right)_{\Omega_{h}}-\left(e_{u}, \nabla \cdot \Pi \boldsymbol{e}_{\boldsymbol{q}}\right)_{\Omega_{h}}+\left\langle\widehat{e}_{u}, \Pi \boldsymbol{e}_{\boldsymbol{q}} \cdot \boldsymbol{n}\right\rangle_{\partial \Omega_{h}}=0,
$$

and hence

$$
\left(\boldsymbol{\Pi} \boldsymbol{e}_{\boldsymbol{q}}, \boldsymbol{\Pi} \boldsymbol{e}_{\boldsymbol{q}}\right)_{\Omega_{h}}-\left(e_{u}, \nabla \cdot \boldsymbol{\Pi} \boldsymbol{e}_{\boldsymbol{q}}\right)_{\Omega_{h}}+\left\langle\widehat{e}_{u}, \boldsymbol{\Pi} \boldsymbol{e}_{\boldsymbol{q}} \cdot \boldsymbol{n}\right\rangle_{\partial \Omega_{h}}=\left(\boldsymbol{\Pi} \boldsymbol{q}-\boldsymbol{q}, \boldsymbol{\Pi} \boldsymbol{e}_{\boldsymbol{q}}\right)_{\Omega_{h}} .
$$

If now we write $e_{u}=\left(I_{h} u-u_{h}\right)-\left(I_{h} u-u\right)$, the above equation becomes

$$
\left\|\boldsymbol{\Pi} \boldsymbol{e}_{\boldsymbol{q}}\right\|_{\boldsymbol{L}^{2}\left(\Omega_{h}\right)}^{2}+T=\left(\boldsymbol{\Pi} \boldsymbol{q}-\boldsymbol{q}, \boldsymbol{\Pi} \boldsymbol{e}_{\boldsymbol{q}}\right)_{\Omega_{h}},
$$

where

$$
T=-\left(I_{h} u-u_{h}, \nabla \cdot \Pi e_{\boldsymbol{q}}\right)_{\Omega_{h}}+\left\langle\widehat{e}_{u}, \Pi e_{\boldsymbol{q}} \cdot \boldsymbol{n}\right\rangle_{\partial \Omega_{h}}+\left(I_{h} u-u, \nabla \cdot \Pi \boldsymbol{e}_{\boldsymbol{q}}\right)_{\Omega_{h}} .
$$

We now simplify $T$. After integration by parts, we get

$$
\begin{aligned}
T= & \left(\boldsymbol{\Pi} \boldsymbol{e}_{\boldsymbol{q}}, \nabla\left(I_{h} u-u_{h}\right)\right)_{\Omega_{h}}-\left\langle\boldsymbol{\Pi} \boldsymbol{e}_{\boldsymbol{q}} \cdot \boldsymbol{n}, I_{h} u-u_{h}-u+\widehat{u}_{h}\right\rangle_{\partial \Omega_{h}} \\
& +\left(I_{h} u-u, \nabla \cdot \boldsymbol{\Pi} \boldsymbol{e}_{\boldsymbol{q}}\right)_{\Omega_{h}},
\end{aligned}
$$

and by the property (3.2a) of the projection $\boldsymbol{\Pi}$,

$$
\begin{aligned}
T= & \left(\boldsymbol{e}_{\boldsymbol{q}}, \nabla\left(I_{h} u-u_{h}\right)\right)_{\Omega_{h}}-\left\langle\boldsymbol{\Pi} \boldsymbol{e}_{\boldsymbol{q}} \cdot \boldsymbol{n}, I_{h} u-u_{h}-u+\widehat{u}_{h}\right\rangle_{\partial \Omega_{h}} \\
& +\left(I_{h} u-u, \nabla \cdot \boldsymbol{\Pi} \boldsymbol{e}_{\boldsymbol{q}}\right)_{\Omega_{h}} .
\end{aligned}
$$

Now, we use the second error equation (3.1b) with $w=I_{h} u-u_{h}$ to obtain

$$
\begin{aligned}
T= & \left\langle\widehat{\boldsymbol{e}}_{\boldsymbol{q}} \cdot \boldsymbol{n}, I_{h} u-u_{h}\right\rangle_{\partial \Omega_{h}}-\left\langle\boldsymbol{\Pi} \boldsymbol{e}_{\boldsymbol{q}} \cdot \boldsymbol{n}, I_{h} u-u_{h}-u+\widehat{u}_{h}\right\rangle_{\partial \Omega_{h}} \\
& +\left(I_{h} u-u, \nabla \cdot \boldsymbol{\Pi} \boldsymbol{e}_{\boldsymbol{q}}\right)_{\Omega_{h}},
\end{aligned}
$$

and rewrite $T$ as $\sum_{i=1}^{4} T_{i}$ where

$$
\begin{aligned}
& T_{1}=\left\langle\widehat{\boldsymbol{e}}_{\boldsymbol{q}} \cdot \boldsymbol{n}, I_{h} u-\widehat{u}_{h}\right\rangle_{\partial \Omega_{h}}, \\
& T_{2}=\left\langle\widehat{\boldsymbol{e}}_{\boldsymbol{q}} \cdot \boldsymbol{n}, \widehat{u}_{h}-u_{h}\right\rangle_{\partial \Omega_{h}}, \\
& T_{3}=\left\langle\boldsymbol{\Pi} \boldsymbol{e}_{\boldsymbol{q}} \cdot \boldsymbol{n}, u_{h}-\widehat{u}_{h}\right\rangle_{\partial \Omega_{h}}, \\
& T_{4}=\left\langle\boldsymbol{\Pi} \boldsymbol{e}_{\boldsymbol{q}} \cdot \boldsymbol{n}, u-I_{h} u\right\rangle_{\partial \Omega_{h}}+\left(I_{h} u-u, \nabla \cdot \boldsymbol{\Pi} \boldsymbol{e}_{\boldsymbol{q}}\right)_{\Omega_{h}} .
\end{aligned}
$$


Let us work on each of these four terms. We begin by noting that, by the third error equation (3.1c) with $\mu:=\left.\left(I_{h} u-\widehat{u}_{h}\right)\right|_{\mathscr{E}_{h}}$, we have that

$$
T_{1}=0 .
$$

Let us work on then term $T_{2}$. By the error equation (3.1d),

$$
T_{2}=\left\langle\boldsymbol{e}_{\boldsymbol{q}} \cdot \boldsymbol{n}, \widehat{u}_{h}-u_{h}\right\rangle_{\partial \Omega_{h}}+\sum_{K \in \Omega_{h}}\left\|\tau^{1 / 2}\left(u_{h}-\widehat{u}_{h}\right)\right\|_{L^{2}(\partial K)}^{2}
$$

and so

$$
\begin{aligned}
T_{2}+T_{3} & =\left\langle(\boldsymbol{q}-\boldsymbol{\Pi} \boldsymbol{q}) \cdot \boldsymbol{n}, \widehat{u}_{h}-u_{h}\right\rangle_{\partial \Omega_{h}}+\sum_{K \in \Omega_{h}}\left\|\tau^{1 / 2}\left(u_{h}-\widehat{u}_{h}\right)\right\|_{L^{2}(\partial K)}^{2}, \\
& =\left\langle\left(\mathbf{P}_{\partial} \boldsymbol{q}-\boldsymbol{\Pi} \boldsymbol{q}\right) \cdot \boldsymbol{n}, \widehat{u}_{h}-u_{h}\right\rangle_{\partial \Omega_{h}}+\sum_{K \in \Omega_{h}}\left\|\tau^{1 / 2}\left(u_{h}-\widehat{u}_{h}\right)\right\|_{L^{2}(\partial K)}^{2},
\end{aligned}
$$

by the definition of the projection $\mathbf{P}_{\partial}$. Finally, after a simple integration by parts, we obtain

$$
T_{4}=\left(\nabla\left(u-I_{h} u\right), \Pi e_{\boldsymbol{q}}\right)_{\Omega_{h}} .
$$

If we substitute the above expressions for $T=\sum_{i=1}^{4} T_{i}$ into the equation (3.3), we get

$$
\begin{aligned}
\Theta:= & \left\|\boldsymbol{\Pi} \boldsymbol{e}_{\boldsymbol{q}}\right\|_{\boldsymbol{L}^{2}\left(\Omega_{h}\right)}^{2}+\sum_{K \in \Omega_{h}}\left\|\tau^{1 / 2}\left(u_{h}-\widehat{u}_{h}\right)\right\|_{L^{2}(\partial K)}^{2} \\
= & \left(\boldsymbol{\Pi} \boldsymbol{q}-\boldsymbol{q}, \boldsymbol{\Pi} \boldsymbol{e}_{\boldsymbol{q}}\right)_{\Omega_{h}}+\left(\nabla\left(I_{h} u-u\right), \boldsymbol{\Pi} \boldsymbol{e}_{\boldsymbol{q}}\right)_{\Omega_{h}} \\
& -\left\langle\left(\boldsymbol{\Pi} \boldsymbol{q}-\mathbf{P}_{\partial} \boldsymbol{q}\right) \cdot \boldsymbol{n}, u_{h}-\widehat{u}_{h}\right\rangle_{\partial \Omega_{h}},
\end{aligned}
$$

and, after a simple application of the Cauchy-Schwarz inequality we get that

$$
\begin{aligned}
\Theta \leq & \|\boldsymbol{\Pi} \boldsymbol{q}-\boldsymbol{q}\|_{\boldsymbol{L}^{2}\left(\Omega_{h}\right)} \Theta^{1 / 2}+\left\|\nabla\left(I_{h} u-u\right)\right\|_{\boldsymbol{L}^{2}\left(\Omega_{h}\right)} \Theta^{1 / 2} \\
& +\left(\sum_{K \in \Omega_{h}} \frac{1}{\tau_{K}}\left\|\left(\boldsymbol{\Pi} \boldsymbol{q}-\mathbf{P}_{\partial} \boldsymbol{q}\right) \cdot \boldsymbol{n}\right\|_{L^{2}\left(e_{K}^{\tau}\right)}^{2}\right)^{1 / 2} \Theta^{1 / 2}
\end{aligned}
$$

and so

$$
\begin{aligned}
\Theta \leq & C\|\boldsymbol{\Pi} \boldsymbol{q}-\boldsymbol{q}\|_{\boldsymbol{L}^{2}\left(\Omega_{h}\right)}^{2}+C\left\|\nabla\left(I_{h} u-u\right)\right\|_{\boldsymbol{L}^{2}\left(\Omega_{h}\right)}^{2} \\
& +C \sum_{K \in \Omega_{h}} \frac{1}{\tau_{K}}\left\|\left(\boldsymbol{\Pi} \boldsymbol{q}-\mathbf{P}_{\partial} \boldsymbol{q}\right) \cdot \boldsymbol{n}\right\|_{L^{2}\left(e_{K}^{\tau}\right)}^{2} .
\end{aligned}
$$

This completes the proof.

Step 2: A second estimate of the error in the flux. Although the sharpness of the estimate we have obtained can be verified numerically, see our numerical experiments, its proof does not shed light on why is it that, unlike what happens for the HDG method, the convergence is suboptimal. Next, we provide an alternative estimate for the error in the flux which tried to remedy this situation; it also holds for 
quite different choices of the local stabilization parameters $\tau$. We follow the approach introduced in [10] which is based on the use of a post-processed approximate flux $\boldsymbol{q}_{h}^{\star}$ we define next.

The function $\boldsymbol{q}_{h}^{\star}$ is constructed from $\boldsymbol{q}_{h}$ and $\widehat{\boldsymbol{q}}_{h}$ as follows. On each simplex $K \in \Omega_{h}$, we define the function $\boldsymbol{q}_{h}^{\star}$ as the only element of $\mathcal{P}_{k}(K)+\boldsymbol{x} \mathcal{P}_{k}(K)$ satisfying

$$
\begin{array}{rlrl}
\left\langle\left(\boldsymbol{q}_{h}^{\star}-\widehat{\boldsymbol{q}}_{h}\right) \cdot \boldsymbol{n}, \mu\right\rangle_{e} & =0 & \forall & \mu \in \mathcal{P}^{k}(e) \text { for all faces } e \text { of } K, \\
\left(\boldsymbol{q}_{h}^{\star}-\boldsymbol{q}_{h}, \boldsymbol{v}\right)_{K} & =0 & \forall \boldsymbol{v} \in \mathcal{P}^{k-1}(K) .
\end{array}
$$

This definition is a modification of that of the Raviart-Thomas projection which is defined as follows. Given a function $\boldsymbol{\sigma} \in \boldsymbol{H}^{1}\left(\Omega_{h}\right)$ and an arbitrary simplex $K \in \Omega_{h}$, the restriction of $\boldsymbol{\Pi}_{\ell}^{\mathrm{RT}} \boldsymbol{\sigma}$ on $K$ is defined as the only element of $\mathcal{P}^{\ell}(K)+\boldsymbol{x} \mathcal{P}^{\ell}(K)$ satisfying

$$
\begin{aligned}
& \left\langle\left(\boldsymbol{\Pi}_{\ell}^{\mathrm{RT}} \boldsymbol{\sigma}-\boldsymbol{\sigma}\right) \cdot \boldsymbol{n}, \mu\right\rangle_{e}=0 \quad \forall \mu \in \mathcal{P}^{\ell}(e) \text { for all faces } e \text { of } K, \\
& \left(\boldsymbol{\Pi}_{\ell}^{\mathrm{RT}} \boldsymbol{\sigma}-\boldsymbol{\sigma}, \boldsymbol{v}\right)_{K}=0 \quad \forall \boldsymbol{v} \in \mathcal{P}^{\ell-1}(K) .
\end{aligned}
$$

We gather the main properties of $\boldsymbol{q}_{h}^{\star}$ in a result similar to that of Lemma 4.1 in [10].

LEMMA 3.3. We have that

(i) $\left(\boldsymbol{q}_{h}^{\star}-\boldsymbol{\Pi}_{k}^{\mathrm{RT}} \boldsymbol{q}\right) \cdot \boldsymbol{n}=0$ on $\partial \Omega_{N}$,

(ii) $\nabla \cdot\left(\boldsymbol{q}_{h}^{\star}-\boldsymbol{\Pi}_{k}^{\mathrm{RT}} \boldsymbol{q}\right)=0$ in $\Omega_{h}$.

(iii) $\boldsymbol{q}_{h}^{\star}-\boldsymbol{\Pi}_{k}^{\mathrm{RT}} \boldsymbol{q} \in \boldsymbol{V}_{h}$.

Next, we use this result to obtain the following auxiliary estimate.

LEMma 3.4. We have

$$
\begin{aligned}
\left\|\boldsymbol{q}_{h}^{\star}-\boldsymbol{\Pi}_{k}^{\mathrm{RT}} \boldsymbol{q}\right\|_{\boldsymbol{L}^{2}\left(\Omega_{h}\right)} \leq & \left\|\boldsymbol{q}-\boldsymbol{\Pi}_{k}^{\mathrm{RT}} \boldsymbol{q}\right\|_{\boldsymbol{L}^{2}\left(\Omega_{h}\right)} \\
& +\left\|\boldsymbol{q}_{h}^{\star}-\boldsymbol{q}_{h}\right\|_{\boldsymbol{L}^{2}\left(\Omega_{h}\right)} \\
& +\left\|\nabla\left(u-I_{h} u\right)\right\|_{\boldsymbol{L}^{2}\left(\Omega_{h}\right)} .
\end{aligned}
$$

Proof. Since, by Property (iii) of Lemma 3.3, we can take $\boldsymbol{v}:=\boldsymbol{\Pi}_{k}^{\mathrm{RT}} \boldsymbol{q}-\boldsymbol{q}_{h}^{\star}$ in the first error equation (3.1a). Using Property (ii), we get

$$
\left(\boldsymbol{e}_{\boldsymbol{q}}, \boldsymbol{\Pi}_{k}^{\mathrm{RT}} \boldsymbol{q}-\boldsymbol{q}_{h}^{\star}\right)_{\Omega_{h}}=-\left\langle\widehat{e}_{u},\left(\boldsymbol{\Pi}_{k}^{\mathrm{RT}} \boldsymbol{q}-\boldsymbol{q}_{h}^{\star}\right) \cdot \boldsymbol{n}\right\rangle_{\partial \Omega_{h}},
$$

or, equivalently,

$$
\begin{aligned}
\left\|\boldsymbol{\Pi}_{k}^{\mathrm{RT}} \boldsymbol{q}-\boldsymbol{q}_{h}^{\star}\right\|_{\boldsymbol{L}^{2}\left(\Omega_{h}\right)}^{2}= & \left(\boldsymbol{\Pi}_{k}^{\mathrm{RT}} \boldsymbol{q}-\boldsymbol{q}, \boldsymbol{q}_{h}^{\star}-\boldsymbol{\Pi}_{k}^{\mathrm{RT}} \boldsymbol{q}\right)_{\Omega_{h}} \\
& -\left(\boldsymbol{q}_{h}^{\star}-\boldsymbol{q}_{h}, \boldsymbol{q}_{h}^{\star}-\boldsymbol{\Pi}_{k}^{\mathrm{RT}} \boldsymbol{q}\right)_{\Omega_{h}} \\
& -\left\langle\widehat{e}_{u},\left(\boldsymbol{q}_{h}^{\star}-\boldsymbol{\Pi}_{k}^{\mathrm{RT}} \boldsymbol{q}\right) \cdot \boldsymbol{n}\right\rangle_{\partial \Omega_{h}} .
\end{aligned}
$$

Since,

$$
\begin{aligned}
-\left\langle\widehat{e}_{u},\left(\boldsymbol{q}_{h}^{\star}-\boldsymbol{\Pi}_{k}^{\mathrm{RT}} \boldsymbol{q}\right) \cdot \boldsymbol{n}\right\rangle_{\partial \Omega_{h}} & =-\left\langle u-\widehat{u}_{h},\left(\boldsymbol{q}_{h}^{\star}-\boldsymbol{\Pi}_{k}^{\mathrm{RT}} \boldsymbol{q}\right) \cdot \boldsymbol{n}\right\rangle_{\partial \Omega_{h}} \\
& =-\left\langle u-I_{h} u,\left(\boldsymbol{q}_{h}^{\star}-\boldsymbol{\Pi}_{k}^{\mathrm{RT}} \boldsymbol{q}\right) \cdot \boldsymbol{n}\right\rangle_{\partial \Omega_{h}}
\end{aligned}
$$

by the error equation (3.1c). Hence,

$$
-\left\langle\widehat{e}_{u},\left(\boldsymbol{q}_{h}^{\star}-\boldsymbol{\Pi}_{k}^{\mathrm{RT}} \boldsymbol{q}\right) \cdot \boldsymbol{n}\right\rangle_{\partial \Omega_{h}}=-\left(\nabla\left(u-I_{h} u\right), \boldsymbol{q}_{h}^{\star}-\boldsymbol{\Pi}_{k}^{\mathrm{RT}} \boldsymbol{q}\right)_{\Omega_{h}},
$$


again by Property (ii) of Lemma 3.3. This implies that

$$
\begin{aligned}
\left\|\boldsymbol{\Pi}_{k}^{\mathrm{RT}} \boldsymbol{q}-\boldsymbol{q}_{h}^{\star}\right\|_{\boldsymbol{L}^{2}\left(\Omega_{h}\right)}^{2}= & \left(\boldsymbol{\Pi}_{k}^{\mathrm{RT}} \boldsymbol{q}-\boldsymbol{q}, \boldsymbol{q}_{h}^{\star}-\boldsymbol{\Pi}_{k}^{\mathrm{RT}} \boldsymbol{q}\right)_{\Omega_{h}} \\
& -\left(\boldsymbol{q}_{h}^{\star}-\boldsymbol{q}_{h}, \boldsymbol{q}_{h}^{\star}-\boldsymbol{\Pi}_{k}^{\mathrm{RT}} \boldsymbol{q}\right)_{\Omega_{h}} \\
& -\left(\nabla\left(u-I_{h} u\right), \boldsymbol{q}_{h}^{\star}-\boldsymbol{\Pi}_{k}^{\mathrm{RT}} \boldsymbol{q}\right)_{\Omega_{h}} .
\end{aligned}
$$

The result now follows after a simple application of Cauchy-Schwarz inequality. This completes the proof. $\square$

Note that in the case of the HDG methods, the estimate of the previous lemma can be improved as follows:

$$
\left\|\boldsymbol{q}_{h}^{\star}-\boldsymbol{\Pi}_{k}^{\mathrm{RT}} \boldsymbol{q}\right\|_{\boldsymbol{L}^{2}\left(\Omega_{h}\right)} \leq\left\|\boldsymbol{q}-\boldsymbol{\Pi}_{k}^{\mathrm{RT}} \boldsymbol{q}\right\|_{\boldsymbol{L}^{2}\left(\Omega_{h}\right)}+\left\|\boldsymbol{q}_{h}^{\star}-\boldsymbol{q}_{h}\right\|_{\boldsymbol{L}^{2}\left(\Omega_{h}\right)} .
$$

Hence, under a suitable choice of the local stabilization parameters $\tau$, the error in the approximate flux is optimal, that is, of order $h^{k+1}$. The reason is that the one of the terms appearing in the previous proof, namely,

$$
-\left\langle\widehat{e}_{u},\left(\boldsymbol{q}_{h}^{\star}-\boldsymbol{\Pi}_{k}^{\mathrm{RT}} \boldsymbol{q}\right) \cdot \boldsymbol{n}\right\rangle_{\partial \Omega_{h}},
$$

is identically equal to zero. However, in our case this is no longer true. This is because on the Dirichlet border, $\widehat{u}_{h}$ is not equal to $\mathrm{P}_{\partial} g$ and, more importantly, because the numerical trace $\widehat{\boldsymbol{q}}_{h} \cdot \boldsymbol{n}$ is not single valued. Indeed, note that

$$
\begin{aligned}
-\left\langle\widehat{e}_{u},\left(\boldsymbol{q}_{h}^{\star}-\boldsymbol{\Pi}_{k}^{\mathrm{RT}} \boldsymbol{q}\right) \cdot \boldsymbol{n}\right\rangle_{\partial \Omega_{h}}= & -\left\langle\mathrm{P}_{\partial} u-\widehat{u}_{h},\left(\widehat{\boldsymbol{q}}_{h}-\boldsymbol{q}\right) \cdot \boldsymbol{n}\right\rangle_{\partial \Omega_{h}} \\
= & -\left\langle\mathrm{P}_{\partial} g-\tilde{I}_{h} g,\left(\widehat{\boldsymbol{q}}_{h}-\boldsymbol{q}\right) \cdot \boldsymbol{n}\right\rangle_{\partial \Omega_{D}} \\
& -\left\langle\mathrm{P}_{\partial} u-I_{h} u, \widehat{\boldsymbol{q}}_{h} \cdot \boldsymbol{n}\right\rangle_{\partial \Omega_{h} \backslash \partial \Omega} .
\end{aligned}
$$

This term gives rise to the last term in the right-hand side of the estimate in Lemma 3.4 and, as we are going to see, ultimately results in the sub-optimality of the convergence of the flux.

The final result in this step is the following.

Corollary 3.5. We have

$$
\begin{aligned}
\left\|\boldsymbol{q}-\boldsymbol{q}_{h}\right\|_{\boldsymbol{L}^{2}\left(\Omega_{h}\right) \leq} & 2\left\|\boldsymbol{q}-\boldsymbol{\Pi}_{k}^{\mathrm{RT}} \boldsymbol{q}\right\|_{\boldsymbol{L}^{2}\left(\Omega_{h}\right)} \\
& +C\left\|\left(\widehat{\boldsymbol{q}}_{h}-\boldsymbol{q}_{h}\right) \cdot \boldsymbol{n}\right\|_{L^{2}\left(\partial \Omega_{h} ; h\right)} \\
& +\left\|\nabla\left(u-I_{h} u\right)\right\|_{\boldsymbol{L}^{2}\left(\Omega_{h}\right)} .
\end{aligned}
$$

Proof. Since we have

$$
\begin{aligned}
\left\|\boldsymbol{q}-\boldsymbol{q}_{h}\right\|_{\boldsymbol{L}^{2}\left(\Omega_{h}\right)} \leq & \left\|\boldsymbol{q}-\boldsymbol{\Pi}_{k}^{\mathrm{RT}} \boldsymbol{q}\right\|_{\boldsymbol{L}^{2}\left(\Omega_{h}\right)} \\
& +\left\|\boldsymbol{\Pi}_{k}^{\mathrm{RT}} \boldsymbol{q}-\boldsymbol{q}_{h}^{\star}\right\|_{\boldsymbol{L}^{2}\left(\Omega_{h}\right)} \\
& +\left\|\boldsymbol{q}_{h}^{\star}-\boldsymbol{q}_{h}\right\|_{\boldsymbol{L}^{2}\left(\Omega_{h}\right)}
\end{aligned}
$$

by Lemma 3.4 we get

$$
\begin{aligned}
\left\|\boldsymbol{q}-\boldsymbol{q}_{h}\right\|_{\boldsymbol{L}^{2}\left(\Omega_{h}\right)} \leq & 2\left\|\boldsymbol{q}-\boldsymbol{\Pi}_{k}^{\mathrm{RT}} \boldsymbol{q}\right\|_{\boldsymbol{L}^{2}\left(\Omega_{h}\right)} \\
& +2\left\|\boldsymbol{q}_{h}^{\star}-\boldsymbol{q}_{h}\right\|_{\boldsymbol{L}^{2}\left(\Omega_{h}\right)} \\
& +\left\|\nabla\left(u-I_{h} u\right)\right\|_{\boldsymbol{L}^{2}\left(\Omega_{h}\right)}
\end{aligned}
$$


The result now follows from the estimate

$$
\left\|\boldsymbol{q}_{h}^{\star}-\boldsymbol{q}_{h}\right\|_{\boldsymbol{L}^{2}\left(\Omega_{h}\right)}^{2} \leq C \sum_{K \in \Omega_{h}} h_{K}\left\|\left(\widehat{\boldsymbol{q}}_{h}-\boldsymbol{q}_{h}\right) \cdot \boldsymbol{n}\right\|_{L^{2}(\partial K)}^{2},
$$

obtained in [10]. This completes the proof.

Step 3:. Next we state a corollary of this lemma.

Corollary 3.6.

$$
\begin{aligned}
\left\|\boldsymbol{q}_{h}-\boldsymbol{q}\right\|_{\boldsymbol{L}^{2}\left(\Omega_{h}\right) \leq} & C\left((1+\rho) h^{k+1}+\rho \kappa h^{k}\right)|\boldsymbol{q}|_{\boldsymbol{H}^{k+1}\left(\Omega_{h}\right)} \\
& +C(1+\rho) h^{k}|u|_{H^{k+1}\left(\Omega_{h}\right)},
\end{aligned}
$$

where the parameter $\rho$ is given by $(2.3 \mathrm{~b})$.

Proof. We have that, by the definition of the numerical trace $\widehat{\boldsymbol{q}}_{h},(1.3 \mathrm{~d})$,

$$
\begin{aligned}
\left\|\left(\widehat{\boldsymbol{q}}_{h}-\boldsymbol{q}_{h}\right) \cdot \boldsymbol{n}\right\|_{L^{2}\left(\partial \Omega_{h} ; h\right)}^{2}= & \sum_{K \in \Omega_{h}} h_{K}\left\|\tau\left(u_{h}-\widehat{u}_{h}\right)\right\|_{L^{2}\left(\partial K \backslash e_{K}^{\tau}\right)}^{2} \\
& +\sum_{K \in \Omega_{h}} h_{K}\left\|\tau\left(u_{h}-\widehat{u}_{h}\right)\right\|_{L^{2}\left(e_{K}^{\tau}\right)}^{2} .
\end{aligned}
$$

Since, by using an argument introduced in [10], see subsection 7.3 therein, we can easily show that

$$
\begin{aligned}
\left\|\tau\left(u_{h}-\widehat{u}_{h}\right)\right\|_{L^{2}\left(e_{K}^{\tau}\right) \leq} & C\left\|\tau\left(u_{h}-\widehat{u}_{h}\right)\right\|_{L^{2}\left(\partial K \backslash e_{K}^{\tau}\right)} \\
& +C\left\|\left(\boldsymbol{\Pi} \boldsymbol{q}-\mathbf{P}_{\partial} \boldsymbol{q}\right) \cdot \boldsymbol{n}\right\|_{L^{2}\left(e_{K}^{\tau}\right)}
\end{aligned}
$$

for all $K \in \Omega_{h}$, we conclude that

$$
\begin{aligned}
\left\|\left(\widehat{\boldsymbol{q}}_{h}-\boldsymbol{q}_{h}\right) \cdot \boldsymbol{n}\right\|_{L^{2}\left(\partial \Omega_{h} ; h\right)}^{2} \leq & C \sum_{K \in \Omega_{h}} h_{K}\left\|\tau\left(u_{h}-\widehat{u}_{h}\right)\right\|_{L^{2}\left(\partial K \backslash e_{K}^{\tau}\right)}^{2} \\
& +C\left(\sum_{K \in \Omega_{h}} h_{K}\left\|\left(\boldsymbol{\Pi} \boldsymbol{q}-\mathbf{P}_{\partial} \boldsymbol{q}\right) \cdot \boldsymbol{n}\right\|_{L^{2}\left(e_{K}^{\tau}\right)}^{2}\right) .
\end{aligned}
$$

Inserting the above estimate in the estimate of Corollary 3.5, we get

$$
\begin{aligned}
\left\|\boldsymbol{q}_{h}-\boldsymbol{q}\right\|_{\boldsymbol{L}^{2}\left(\Omega_{h}\right)} \leq & C\left\|\boldsymbol{q}-\boldsymbol{\Pi}_{k}^{\mathrm{RT}} \boldsymbol{q}\right\|_{\boldsymbol{L}^{2}\left(\Omega_{h}\right)} \\
& +C \rho\left(\sum_{K \in \Omega_{h}}\left\|\tau^{1 / 2}\left(u_{h}-\widehat{u}_{h}\right)\right\|_{L^{2}(\partial K)}^{2}\right)^{1 / 2} \\
& +C\left(\sum_{K \in \Omega_{h}} h_{K}\left\|\left(\boldsymbol{\Pi} \boldsymbol{q}-\mathbf{P}_{\partial} \boldsymbol{q}\right) \cdot \boldsymbol{n}\right\|_{L^{2}\left(e_{K}^{\tau}\right)}^{2}\right)^{1 / 2} \\
& +\left\|\nabla\left(u-I_{h} u\right)\right\|_{\boldsymbol{L}^{2}\left(\Omega_{h}\right)},
\end{aligned}
$$

and the result follows by using the definition of $\rho,(2.3 \mathrm{~b})$, and approximation results for $\Pi$ and $I_{h}$. This completes the proof.

Step 4: Conclusion. The estimate of Theorem 2.1 follows by combining the estimate Corollary 3.2 with that of Corollary 3.6. This completes the proof of Theorem 2.1 . 
3.3. Proof of the estimates for the error in $u$. Here, we devote ourselves to proving Theorems 2.2 and 2.3.

Proof of the estimate for $u-u_{h}$, Theorem 2.2. To prove the estimate of Theorem 2.2, we first state an auxiliary lemma. To state it, we need to define a projection that was first introduced [7] and later used in [6]. For any function $u \in H^{1}\left(\Omega_{h}\right)$, the projection $\mathbb{P} u \in W_{h}$ is defined on each $K \in \Omega_{h}$ and satisfies the following equations

$$
\begin{aligned}
(\mathbb{P} u-u, \omega)_{K} & =0, \quad \forall \quad \omega \in \mathcal{P}^{k-1}(K) \\
\langle\mathbb{P} u-u, \mu\rangle_{e_{K}^{\tau}} & =0, \quad \forall \quad \mu \in \mathcal{P}^{k}\left(e_{K}^{\tau}\right) .
\end{aligned}
$$

We are now ready to state the result.

Lemma 3.7. We have

$$
\begin{aligned}
\left\|u-u_{h}\right\|_{L^{2}\left(\Omega_{h}\right) \leq} & C h\left\|\boldsymbol{q}-\boldsymbol{q}_{h}\right\|_{\boldsymbol{L}^{2}\left(\Omega_{h}\right)}+\left\|g-\tilde{I}_{h} g\right\|_{L^{2}\left(\partial \Omega_{D}\right)} \\
& +C \kappa\left(\sum_{K \in \Omega_{h}}\left\|\tau^{1 / 2}\left(u_{h}-\widehat{u}_{h}\right)\right\|_{L^{2}\left(e_{K}^{\tau}\right)}^{2}\right)^{1 / 2}+\|\mathbb{P} u-u\|_{L^{2}\left(\Omega_{h}\right)} .
\end{aligned}
$$

Theorem 2.2 follows from the above lemma, Corollary 3.2 and the fact that

$$
\|\mathbb{P} u-u\|_{L^{2}\left(\Omega_{h}\right)} \leq C h^{k+1}\|u\|_{H^{k+1}\left(\Omega_{h}\right)} .
$$

Finally, we prove Lemma 3.7.

Proof. If $(\boldsymbol{\psi}, \sigma)$ is the solution of the dual problem (2.4) with $\theta:=\mathbb{P} u-u_{h}$, we can write

$$
\begin{aligned}
\left\|\mathbb{P} u-u_{h}\right\|_{L^{2}\left(\Omega_{h}\right)}^{2} & =\left(\mathbb{P} e_{u}, \nabla \cdot \boldsymbol{\psi}\right)_{\Omega_{h}} \\
& =\left(\mathbb{P} e_{u}, \nabla \cdot(\boldsymbol{\psi}-\Pi \boldsymbol{\Pi})\right)_{\Omega_{h}}+\left(e_{u}, \nabla \cdot \Pi \boldsymbol{\Pi}\right)_{\Omega_{h}},
\end{aligned}
$$

by the property (3.6a) of the projection $\mathbb{P}$. Now, by the property (3.2a) of the projection $\Pi$, we have

$$
\left\|\mathbb{P} u-u_{h}\right\|_{L^{2}\left(\Omega_{h}\right)}^{2}=\left\langle\mathbb{P} e_{u},(\boldsymbol{\psi}-\boldsymbol{\Pi} \boldsymbol{\psi}) \cdot \boldsymbol{n}\right\rangle_{\partial \Omega_{h}}+\left(e_{u}, \nabla \cdot \boldsymbol{\Pi} \boldsymbol{\psi}\right)_{\Omega_{h}},
$$

and, by the error equation (3.1a) with $\boldsymbol{v}:=\boldsymbol{\Pi} \boldsymbol{\psi}$,

$$
\begin{aligned}
\left\|\mathbb{P} u-u_{h}\right\|_{L^{2}\left(\Omega_{h}\right)}^{2} & =\left\langle\mathbb{P} e_{u},(\boldsymbol{\psi}-\boldsymbol{\Pi} \boldsymbol{\psi}) \cdot \boldsymbol{n}\right\rangle_{\partial \Omega_{h}}+\left(\boldsymbol{e}_{\boldsymbol{q}}, \boldsymbol{\Pi} \boldsymbol{\psi}\right)_{\Omega_{h}}+\left\langle\widehat{e}_{u}, \boldsymbol{\Pi} \boldsymbol{\psi} \cdot \boldsymbol{n}\right\rangle_{\partial \Omega_{h}} \\
& =\left(\boldsymbol{e}_{\boldsymbol{q}}, \boldsymbol{\Pi} \boldsymbol{\psi}\right)_{\Omega_{h}}+T
\end{aligned}
$$

where

$$
T=\left\langle\mathbb{P} e_{u},(\boldsymbol{\psi}-\boldsymbol{\Pi} \boldsymbol{\psi}) \cdot \boldsymbol{n}\right\rangle_{\partial \Omega_{h}}+\left\langle\mathrm{P}_{\partial} \widehat{e}_{u}, \boldsymbol{\Pi} \boldsymbol{\psi} \cdot \boldsymbol{n}\right\rangle_{\partial \Omega_{h}} .
$$

But,

$$
T=\left\langle\mathbb{P} e_{u}-\mathrm{P}_{\partial} \widehat{e}_{u},(\boldsymbol{\psi}-\boldsymbol{\Pi} \boldsymbol{\psi}) \cdot \boldsymbol{n}\right\rangle_{\partial \Omega_{h}}+\left\langle\mathrm{P}_{\partial} \widehat{e}_{u}, \boldsymbol{\psi} \cdot \boldsymbol{n}\right\rangle_{\partial \Omega_{h}}
$$

by definition of $\mathrm{P}_{\partial}$, and so

$$
\begin{aligned}
T= & \left\langle\mathbb{P} u-\mathrm{P}_{\partial} u,(\boldsymbol{\psi}-\boldsymbol{\Pi} \boldsymbol{\psi}) \cdot \boldsymbol{n}\right\rangle_{\partial \Omega_{h}}+\left\langle\widehat{u}_{h}-u_{h},(\boldsymbol{\psi}-\boldsymbol{\Pi} \boldsymbol{\psi}) \cdot \boldsymbol{n}\right\rangle_{\partial \Omega_{h}} \\
& +\left\langle\mathrm{P}_{\partial} g-I_{h} g, \boldsymbol{\psi} \cdot \boldsymbol{n}\right\rangle_{\partial \Omega_{D}},
\end{aligned}
$$


since $\boldsymbol{\psi} \cdot \boldsymbol{n}=0$ on $\partial \Omega_{N}$. Finally, we get that

$$
T=\left\langle\widehat{u}_{h}-u_{h},(\boldsymbol{\psi}-\boldsymbol{\Pi} \boldsymbol{\psi}) \cdot \boldsymbol{n}\right\rangle_{\partial \Omega_{h}}+\left\langle\mathrm{P}_{\partial} g-I_{h} g, \boldsymbol{\psi} \cdot \boldsymbol{n}\right\rangle_{\partial \Omega_{D}},
$$

by Property (iii) in Proposition 2.1 in [7]. Hence, we have

$$
\begin{aligned}
\left\|\mathbb{P} u-u_{h}\right\|_{L^{2}\left(\Omega_{h}\right)}^{2}= & \left(\boldsymbol{e}_{\boldsymbol{q}}, \boldsymbol{\Pi} \boldsymbol{\psi}\right)_{\Omega_{h}}+\left\langle\widehat{u}_{h}-u_{h},\left(\mathbf{P}_{\partial} \boldsymbol{\psi}-\boldsymbol{\Pi} \boldsymbol{\psi}\right) \cdot \boldsymbol{n}\right\rangle_{\partial \Omega_{h}} \\
& +\left\langle\mathrm{P}_{\partial} g-I_{h} g, \boldsymbol{\psi} \cdot \boldsymbol{n}\right\rangle_{\partial \Omega_{D}} .
\end{aligned}
$$

Let us now estimate the first term on the right-hand side. We have

$$
\begin{aligned}
\left(\boldsymbol{e}_{\boldsymbol{q}}, \boldsymbol{\Pi} \boldsymbol{\psi}\right)_{\Omega_{h}} & =\left(\boldsymbol{e}_{\boldsymbol{q}}, \boldsymbol{\psi}\right)_{\Omega_{h}}+\left(\boldsymbol{e}_{\boldsymbol{q}}, \boldsymbol{\Pi} \boldsymbol{\psi}-\boldsymbol{\psi}\right)_{\Omega_{h}} \\
& =-\left(\boldsymbol{e}_{\boldsymbol{q}}, \nabla \sigma\right)_{\Omega_{h}}+\left(\boldsymbol{e}_{\boldsymbol{q}}, \boldsymbol{\Pi} \boldsymbol{\psi}-\boldsymbol{\psi}\right)_{\Omega_{h}} \\
& =-\left(\boldsymbol{e}_{\boldsymbol{q}}, \nabla\left(\sigma-I_{h} \sigma\right)\right)_{\Omega_{h}}+\left(\boldsymbol{e}_{\boldsymbol{q}}, \boldsymbol{\Pi} \boldsymbol{\psi}-\boldsymbol{\psi}\right)_{\Omega_{h}},
\end{aligned}
$$

since $\left(\boldsymbol{e}_{\boldsymbol{q}}, \nabla I_{h} \sigma\right)_{\Omega_{h}}=0$ by the error equations (3.1b) with $\omega:=I_{h} \sigma$ and (3.1c) with $\mu:=I_{h} \sigma$.

Therefore,

$$
\begin{aligned}
\left\|\mathbb{P} u-u_{h}\right\|_{L^{2}\left(\Omega_{h}\right)}^{2}= & -\left(\boldsymbol{e}_{\boldsymbol{q}}, \nabla\left(\sigma-I_{h} \sigma\right)\right)_{\Omega_{h}}+\left(\boldsymbol{e}_{\boldsymbol{q}}, \boldsymbol{\Pi} \boldsymbol{\psi}-\boldsymbol{\psi}\right)_{\Omega_{h}} \\
& +\left\langle\widehat{u}_{h}-u_{h},\left(\mathbf{P}_{\partial} \boldsymbol{\psi}-\boldsymbol{\Pi} \boldsymbol{\psi}\right) \cdot \boldsymbol{n}\right\rangle_{\partial \Omega_{h}}+\left\langle\mathrm{P}_{\partial} g-I_{h} g, \boldsymbol{\psi} \cdot \boldsymbol{n}\right\rangle_{\partial \Omega_{D}} .
\end{aligned}
$$

If we use the Cauchy-Schwarz inequality and the definition of $\kappa$ we get

$$
\begin{aligned}
\left\|\mathbb{P} u-u_{h}\right\|_{L^{2}\left(\Omega_{h}\right)}^{2} \leq & \left\|\boldsymbol{e}_{\boldsymbol{q}}\right\|_{\boldsymbol{L}^{2}\left(\Omega_{h}\right)}\left(\left\|\sigma-I_{h} \sigma\right\|_{L^{2}\left(\Omega_{h}\right)}+\|\boldsymbol{\Pi} \boldsymbol{\psi}-\boldsymbol{\psi}\|_{\boldsymbol{L}^{2}\left(\Omega_{h}\right)}\right) \\
+ & \kappa\left(\sum_{K \in \Omega_{h}}\left\|\tau_{K}^{1 / 2}\left(\widehat{u}_{h}-u_{h}\right)\right\|_{L^{2}\left(e_{K}^{\tau}\right)}^{2}\right)^{1 / 2} \times \\
& \left(\sum_{K \in \Omega_{h}} \frac{1}{h_{K}}\left\|\left(\mathbf{P}_{\partial} \boldsymbol{\psi}-\boldsymbol{\Pi} \boldsymbol{\psi}\right) \cdot \boldsymbol{n}\right\|_{L^{2}\left(e_{K}^{\tau}\right)}^{2}\right)^{1 / 2} \\
+ & \left\|g-\tilde{I}_{h} g\right\|_{L^{2}\left(\partial \Omega_{D}\right)}\|\boldsymbol{\psi}\|_{L^{2}\left(\partial \Omega_{D}\right)}
\end{aligned}
$$

If we use the trace inequality $\|\boldsymbol{\psi}\|_{L^{2}(\partial \Omega)} \leq C\|\boldsymbol{\psi}\|_{\boldsymbol{H}^{1}(\Omega)}$ and approximation properties of $I_{h}$ and $\Pi$ we get

$$
\begin{aligned}
\left\|\mathbb{P} u-u_{h}\right\|_{L^{2}\left(\Omega_{h}\right)}^{2} \leq & C h\left\|\boldsymbol{e}_{\boldsymbol{q}}\right\|_{\boldsymbol{L}^{2}\left(\Omega_{h}\right)}\left(\|\sigma\|_{H^{2}\left(\Omega_{h}\right)}+\|\boldsymbol{\psi}\|_{\boldsymbol{H}^{1}\left(\Omega_{h}\right)}\right) \\
& +C \kappa\left(\sum_{K \in \Omega_{h}}\left\|\tau_{K}^{1 / 2}\left(\widehat{u}_{h}-u_{h}\right)\right\|_{L^{2}\left(e_{K}^{\tau}\right)}^{2}\right)^{1 / 2}\|\boldsymbol{\psi}\|_{\boldsymbol{H}^{1}\left(\Omega_{h}\right)} \\
& +C\left\|g-\tilde{I}_{h} g\right\|_{L^{2}\left(\partial \Omega_{D}\right)}\|\boldsymbol{\psi}\|_{\boldsymbol{H}^{1}(\Omega)} .
\end{aligned}
$$

Hence, after we use the regularity result (2.5), we obtain

$$
\begin{aligned}
\left\|\mathbb{P} u-u_{h}\right\|_{L^{2}\left(\Omega_{h}\right)} \leq & C h\left\|\boldsymbol{q}-\boldsymbol{q}_{h}\right\|_{\boldsymbol{L}^{2}\left(\Omega_{h}\right)}+\left\|g-\tilde{I}_{h} g\right\|_{L^{2}\left(\partial \Omega_{D}\right)} \\
& +C \kappa\left(\sum_{K \in \Omega_{h}}\left\|\tau^{1 / 2}\left(u_{h}-\widehat{u}_{h}\right)\right\|_{L^{2}\left(e_{K}^{\tau}\right)}^{2}\right)^{1 / 2} .
\end{aligned}
$$

The result now follows after using the triangle inequality

$$
\left\|u-u_{h}\right\|_{L^{2}\left(\Omega_{h}\right)} \leq\left\|\mathbb{P} u-u_{h}\right\|_{L^{2}\left(\Omega_{h}\right)}+\|\mathbb{P} u-u\|_{L^{2}\left(\Omega_{h}\right)} .
$$

This completes the proof. $\square$ 
Proofs of the estimate of $\mathrm{P}^{k-1}\left(u-u_{h}\right)$ and $\mathrm{P}_{\partial} u-\widehat{u}_{h}$, Theorem 2.3. Here we are going to need the so-called Brezzi-Douglas-Marini projection, [2]. Let us recall its definition. For any function $\boldsymbol{q} \in \boldsymbol{H}^{1}\left(\Omega_{h}\right)$, and for each simplex $K \in \Omega_{h}$, we define the function $\boldsymbol{\Pi}_{k}^{\mathrm{BDM}} \boldsymbol{q}$ as

$$
\begin{aligned}
\left\langle\left(\boldsymbol{\Pi}_{k}^{\mathrm{BDM}} \boldsymbol{q}-\boldsymbol{q}\right) \cdot \boldsymbol{n}, \mu\right\rangle_{e} & =0 & & \forall \mu \in \mathcal{P}^{k}(e) \text { for all faces } e \text { of } K, \\
\left(\boldsymbol{\Pi}_{k}^{\mathrm{BD}} \boldsymbol{q}-\boldsymbol{q}, \nabla w\right)_{K} & =0 & & \forall w \in \mathcal{P}^{k-1}(K) \\
\left(\boldsymbol{\Pi}_{k}^{\mathrm{BDM}} \boldsymbol{q}-\boldsymbol{q}, \boldsymbol{v}\right)_{K} & =0, & & \forall \boldsymbol{v} \in \mathbf{\Phi}_{k}(K),
\end{aligned}
$$

where

$$
\boldsymbol{\Phi}_{k}(K)=\left\{\boldsymbol{v} \in \mathcal{P}^{k}(K): \nabla \cdot \boldsymbol{v}=0,\left.\boldsymbol{v} \cdot \boldsymbol{n}\right|_{\partial K}=0\right\} .
$$

To prove the first estimate, we take $(\boldsymbol{\psi}, \sigma)$ as the solution of the dual problem (2.4) with $\theta=\mathrm{P}^{k-1}\left(u-u_{h}\right)$ which is the $L^{2}$-projection of $u-u_{h}$ into polynomials of degree $k-1$. Then,

$$
\begin{aligned}
\left\|\mathrm{P}^{k-1}\left(u-u_{h}\right)\right\|_{L^{2}\left(\Omega_{h}\right)}^{2} & =\left(\mathrm{P}^{k-1} e_{u}, \nabla \cdot \boldsymbol{\psi}\right)_{\Omega_{h}} \\
& =\left(\mathrm{P}^{k-1} e_{u}, \nabla \cdot \boldsymbol{\Pi}_{k}^{\mathrm{BDM}} \boldsymbol{\psi}\right)_{\Omega_{h}} \\
& =\left(e_{u}, \nabla \cdot \boldsymbol{\Pi}_{k}^{\mathrm{BDM}} \boldsymbol{\psi}\right)_{\Omega_{h}},
\end{aligned}
$$

by the property (3.7b) of the projection $\boldsymbol{\Pi}_{k}^{\text {BDM }}$. Now, by the error equation (3.1a) with $\boldsymbol{v}:=\boldsymbol{\Pi}_{k}^{\mathrm{BDM}} \boldsymbol{\psi}$, we get

$$
\begin{aligned}
\left\|\mathrm{P}^{k-1}\left(u-u_{h}\right)\right\|_{L^{2}\left(\Omega_{h}\right)}^{2} & =\left(\boldsymbol{e}_{\boldsymbol{q}}, \boldsymbol{\Pi}_{k}^{\mathrm{BDM}} \boldsymbol{\psi}\right)_{\Omega_{h}}+\left\langle u-\widehat{u}_{h}, \boldsymbol{\Pi}_{k}^{\mathrm{BDM}} \boldsymbol{\psi} \cdot \boldsymbol{n}\right\rangle_{\partial \Omega_{h}} \\
& =\left(\boldsymbol{e}_{\boldsymbol{q}}, \boldsymbol{\Pi}_{k}^{\mathrm{BDM}} \boldsymbol{\psi}\right)_{\Omega_{h}}+\left\langle u-\widehat{u}_{h}, \boldsymbol{\Pi}_{k}^{\mathrm{BDM}} \boldsymbol{\psi} \cdot \boldsymbol{n}\right\rangle_{\partial \Omega},
\end{aligned}
$$

since $u, \widehat{u}_{h}$, and the normal component of $\Pi_{k}^{\mathrm{BDM}} \boldsymbol{\psi}$ are single-valued functions on $\mathscr{E}_{h}$. By the Dirichlet boundary condition on $u(1.1 \mathrm{c})$ and on $\widehat{u}_{h}(2.1 \mathrm{e})$, and by the Neumann boundary condition of the dual problem for $\boldsymbol{\psi}(2.4 \mathrm{~d})$, we obtain that

$$
\left\|\mathrm{P}^{k-1}\left(u-u_{h}\right)\right\|_{L^{2}\left(\Omega_{h}\right)}^{2}=\left(\boldsymbol{e}_{\boldsymbol{q}}, \boldsymbol{\Pi}_{k}^{\mathrm{BDM}} \boldsymbol{\psi}\right)_{\Omega_{h}}+\left\langle g-\tilde{I}_{h} g, \boldsymbol{\Pi}_{k}^{\mathrm{BDM}} \boldsymbol{\psi} \cdot \boldsymbol{n}\right\rangle_{\partial \Omega_{D}} .
$$

Finally, proceeding as in the previous proof, we can easily obtain that

$$
\begin{aligned}
\left\|\mathrm{P}^{k-1}\left(u-u_{h}\right)\right\|_{L^{2}\left(\Omega_{h}\right)}^{2}= & -\left(\boldsymbol{e}_{\boldsymbol{q}}, \nabla\left(\sigma-I_{h} \sigma\right)\right)_{\Omega_{h}}+\left(\boldsymbol{e}_{\boldsymbol{q}}, \boldsymbol{\Pi}_{k}^{\mathrm{BDM}} \boldsymbol{\psi}-\boldsymbol{\psi}\right)_{\Omega_{h}} \\
& +\left\langle\mathrm{P}_{\partial} g-\tilde{I}_{h} g, \boldsymbol{\psi} \cdot \boldsymbol{n}\right\rangle_{\partial \Omega_{D}} .
\end{aligned}
$$

The proof of the first estimate of Theorem 2.3 now follows.

To prove the remaining estimate, we proceed as follows. Let $K \in \Omega_{h}$ and define the function $\boldsymbol{\eta} \in \mathcal{P}^{k}(K)$ satisfying

$$
\begin{aligned}
\left\langle\boldsymbol{\eta} \cdot \boldsymbol{n}-\left(\mathrm{P}_{\partial} u-\widehat{u}_{h}\right), \mu\right\rangle_{e} & =0, \quad \forall \mu \in \mathcal{P}^{k}(e), \text { for all faces } e \text { of } \partial K \\
(\boldsymbol{\eta}, \nabla \omega)_{K} & =0, \quad \forall \omega \in \mathcal{P}^{k-1}(K), \\
(\boldsymbol{\eta}, \boldsymbol{v})_{K} & =0, \quad \forall \boldsymbol{v} \in \Phi_{k}(K) .
\end{aligned}
$$

It can be easily seen via a scaling argument that

$$
\|\boldsymbol{\eta}\|_{L^{2}(K)}+h_{K}\|\boldsymbol{\eta}\|_{\boldsymbol{H}^{1}(K)} \leq C h_{K}^{1 / 2}\left\|\mathrm{P}_{\partial} u-\widehat{u}_{h}\right\|_{L^{2}(\partial K)} .
$$


Then

$$
\left\|\mathrm{P}_{\partial} u-\widehat{u}_{h}\right\|_{L^{2}(\partial K)}^{2}=\left\langle\mathrm{P}_{\partial} u-\widehat{u}_{h}, \boldsymbol{\eta} \cdot \boldsymbol{n}\right\rangle_{\partial K}=\left\langle\widehat{e}_{u}, \boldsymbol{\eta} \cdot \boldsymbol{n}\right\rangle_{\partial K},
$$

and by the error equation (3.1a) with $\boldsymbol{v}:=\boldsymbol{\eta}$,

$$
\begin{aligned}
\left\|\mathrm{P}_{\partial} u-\widehat{u}_{h}\right\|_{L^{2}(\partial K)}^{2} & =-\left(\boldsymbol{e}_{\boldsymbol{q}}, \boldsymbol{\eta}\right)_{K}+\left(e_{u}, \nabla \cdot \boldsymbol{\eta}\right)_{K} \\
& =-\left(\boldsymbol{e}_{\boldsymbol{q}}, \boldsymbol{\eta}\right)_{K}+\left(\mathrm{P}^{k-1} e_{u}, \nabla \cdot \boldsymbol{\eta}\right)_{K},
\end{aligned}
$$

and so,

$$
h_{K}\left\|\mathrm{P}_{\partial} u-\widehat{u}_{h}\right\|_{L^{2}(\partial K)}^{2} \leq C h_{K}^{2}\left\|\boldsymbol{e}_{\boldsymbol{q}}\right\|_{\boldsymbol{L}^{2}(K)}^{2}+C\left\|\mathrm{P}^{k-1} e_{u}\right\|_{L^{2}(K)}^{2} .
$$

Therefore, taking the sum over $K \in \Omega_{h}$

$$
\begin{aligned}
\left\|\mathrm{P}_{\partial} u-\widehat{u}_{h}\right\|_{L^{2}\left(\partial \Omega_{h} ; h\right)} & \leq C h\left\|\boldsymbol{e}_{\boldsymbol{q}}\right\|_{\boldsymbol{L}^{2}\left(\Omega_{h}\right)}+C\left\|\mathrm{P}^{k-1} e_{u}\right\|_{L^{2}\left(\Omega_{h}\right)} \\
& \leq C h\left\|\boldsymbol{e}_{\boldsymbol{q}}\right\|_{\boldsymbol{L}^{2}\left(\Omega_{h}\right)}+\left\|\mathrm{P}_{\partial} g-\tilde{I}_{h} g\right\|_{L^{2}\left(\partial \Omega_{D}\right)} .
\end{aligned}
$$

Note that to obtain the last inequality, we used the first inequality of Theorem 2.3. The proof of Theorem of 2.3 is now complete.

4. Numerical results. In this section, we provide numerical experiments validating our theoretical results. To do that, we consider the test problem (1.1), where $\Omega=(0,1) \times(0,1)$, and $u_{D}$ and $f$ are taken in such a way that $u(x, y)=$ $\sin (\pi x) \sin (\pi y)$ is the exact solution; we also take $\partial \Omega_{N}=\emptyset$.

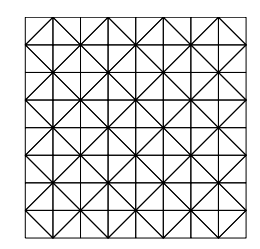

FIG. 4.1. Example of a mesh.

We consider the HDG and EDG methods for which $\tau$ is a constant on $\mathscr{E}_{h}$ and study the convergence properties of the error in the flux $\left\|\boldsymbol{q}-\boldsymbol{q}_{h}\right\|_{\boldsymbol{L}^{2}\left(\Omega_{h}\right)}$ and the error in the scalar variable $\left\|u-u_{h}^{\star}\right\|_{L^{2}\left(\Omega_{h}\right)}$, where $u_{h}^{\star}$ is an approximation to $u$ defined as follows; see $[7,10]$. On the simplex $K, u_{h}^{\star}$, is the function of $\mathcal{P}^{k+1}(K)$ given by

$$
u_{h}^{\star}=\widetilde{u}_{h}+\frac{1}{|K|} \int_{K} u_{h} d x
$$

where $\widetilde{u}_{h}$ is the polynomial in $\mathcal{P}_{0}^{k+1}(K)$ satisfying

$$
\left(\nabla \widetilde{u}_{h}, \nabla w\right)_{K}=(f, w)_{K}-\left\langle w, \widehat{\boldsymbol{q}}_{h} \cdot \boldsymbol{n}\right\rangle_{\partial K} \quad \forall w \in \mathcal{P}_{0}^{k+1}(K) .
$$

Here $\mathcal{P}_{0}^{k+1}(K)$ is the set of polynomials in $\mathcal{P}^{k+1}(K)$ with zero mean. The meshes we take are made of $2^{\ell+1}$ congruent triangles; see an example in Fig. 4.1. 
TABLE 4.1

History of convergence

\begin{tabular}{|c|c|c|c|c|c|c|c|c|c|}
\hline & & \multicolumn{4}{|c|}{ HDG method } & \multicolumn{4}{|c|}{ EDG method } \\
\hline & & \multicolumn{2}{|c|}{$\left\|\boldsymbol{q}-\boldsymbol{q}_{h}\right\|_{L^{2}\left(\Omega_{h}\right)}$} & \multicolumn{2}{|c|}{$\left\|u-u_{h}^{\star}\right\|_{L^{2}\left(\Omega_{h}\right)}$} & \multicolumn{2}{|c|}{$\left\|\boldsymbol{q}-\boldsymbol{q}_{h}\right\|_{L^{2}\left(\Omega_{h}\right)}$} & \multicolumn{2}{|c|}{$\left\|u-u_{h}^{\star}\right\|_{L^{2}\left(\Omega_{h}\right)}$} \\
\hline$k$ & $\ell$ & error & order & error & order & error & or der & error & order \\
\hline \multicolumn{10}{|c|}{$\tau=h$} \\
\hline \multirow{4}{*}{1} & 1 & $9.76 \mathrm{E}-02$ & - & $3.16 \mathrm{E}-03$ & 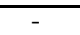 & $7.18 \mathrm{E}-01$ & - & $6.18 \mathrm{E}-02$ & \\
\hline & 2 & $2.45 \mathrm{E}-02$ & 1.99 & $3.82 \mathrm{E}-04$ & 3.05 & $3.68 \mathrm{E}-01$ & 0.97 & $1.60 \mathrm{E}-02$ & 1.95 \\
\hline & 3 & $6.14 \mathrm{E}-03$ & 2.00 & $4.73 \mathrm{E}-05$ & 3.01 & $1.85 \mathrm{E}-01$ & 0.99 & $4.05 \mathrm{E}-03$ & 1.99 \\
\hline & 4 & $1.53 \mathrm{E}-03$ & 2.00 & $5.91 \mathrm{E}-06$ & 3.00 & $9.26 \mathrm{E}-02$ & 1.00 & $1.02 \mathrm{E}-03$ & 2.00 \\
\hline \multirow{4}{*}{2} & 1 & $1.07 \mathrm{E}-02$ & - & $2.96 \mathrm{E}-04$ & - & $9.87 \mathrm{E}-02$ & - & $3.39 \mathrm{E}-03$ & - \\
\hline & 2 & $1.35 \mathrm{E}-03$ & 2.99 & $1.86 \mathrm{E}-05$ & 3.99 & $2.65 \mathrm{E}-02$ & 1.90 & $4.75 \mathrm{E}-04$ & 2.84 \\
\hline & 3 & $1.70 \mathrm{E}-04$ & 3.00 & $1.16 \mathrm{E}-06$ & 4.00 & $6.77 \mathrm{E}-03$ & 1.97 & $6.15 \mathrm{E}-05$ & 2.95 \\
\hline & 4 & $2.12 \mathrm{E}-05$ & 3.00 & $7.26 \mathrm{E}-08$ & 4.00 & $1.70 \mathrm{E}-03$ & 1.99 & $7.75 \mathrm{E}-06$ & 2.99 \\
\hline \multirow{4}{*}{3} & 1 & $9.34 \mathrm{E}-04$ & - & $2.11 \mathrm{E}-05$ & - & $1.05 \mathrm{E}-02$ & - & $2.35 \mathrm{E}-04$ & - \\
\hline & 2 & $5.89 \mathrm{E}-05$ & 3.99 & $6.66 \mathrm{E}-07$ & 4.98 & $1.31 \mathrm{E}-03$ & 3.00 & $1.42 \mathrm{E}-05$ & 4.05 \\
\hline & 3 & $3.69 \mathrm{E}-06$ & 4.00 & $2.09 \mathrm{E}-08$ & 5.00 & $1.64 \mathrm{E}-04$ & 3.00 & $8.77 \mathrm{E}-07$ & 4.02 \\
\hline & 4 & $2.31 \mathrm{E}-07$ & 4.00 & $6.68 \mathrm{E}-10$ & 4.97 & $2.05 \mathrm{E}-05$ & 3.00 & $5.46 \mathrm{E}-08$ & 4.00 \\
\hline \multicolumn{10}{|c|}{$\tau=1$} \\
\hline \multirow{4}{*}{1} & 1 & $1.01 \mathrm{E}-01$ & - & $3.86 \mathrm{E}-03$ & - & $7.18 \mathrm{E}-01$ & - & $6.21 \mathrm{E}-02$ & - \\
\hline & 2 & $2.55 \mathrm{E}-02$ & 1.99 & $4.73 \mathrm{E}-04$ & 3.03 & $3.68 \mathrm{E}-01$ & 0.97 & $1.61 \mathrm{E}-02$ & 1.95 \\
\hline & 3 & $6.38 \mathrm{E}-03$ & 2.00 & $5.86 \mathrm{E}-05$ & 3.01 & $1.85 \mathrm{E}-01$ & 0.99 & $4.06 \mathrm{E}-03$ & 1.99 \\
\hline & 4 & $1.59 \mathrm{E}-03$ & 2.00 & $7.29 \mathrm{E}-06$ & 3.01 & $9.26 \mathrm{E}-02$ & 1.00 & $1.02 \mathrm{E}-03$ & 2.00 \\
\hline & 1 & $1.11 \mathrm{E}-02$ & - & $3.03 \mathrm{E}-04$ & - & $9.88 \mathrm{E}-02$ & - & $3.39 \mathrm{E}-03$ & - \\
\hline \multirow{3}{*}{2} & 2 & $1.41 \mathrm{E}-03$ & 2.99 & $1.90 \mathrm{E}-05$ & 4.00 & $2.65 \mathrm{E}-02$ & 1.90 & $4.75 \mathrm{E}-04$ & 2.84 \\
\hline & 3 & $1.76 \mathrm{E}-04$ & 3.00 & $1.18 \mathrm{E}-06$ & 4.00 & $6.77 \mathrm{E}-03$ & 1.97 & $6.14 \mathrm{E}-05$ & 2.95 \\
\hline & 4 & $2.20 \mathrm{E}-05$ & 3.00 & $7.37 \mathrm{E}-08$ & 4.00 & $1.70 \mathrm{E}-03$ & 1.99 & $7.75 \mathrm{E}-06$ & 2.99 \\
\hline \multirow{4}{*}{3} & 1 & $9.69 \mathrm{E}-04$ & - & $2.11 \mathrm{E}-05$ & - & $1.06 \mathrm{E}-02$ & - & $2.34 \mathrm{E}-04$ & \\
\hline & 2 & $6.11 \mathrm{E}-05$ & 3.99 & $6.68 \mathrm{E}-07$ & 4.98 & $1.31 \mathrm{E}-03$ & 3.00 & $1.42 \mathrm{E}-05$ & 4.05 \\
\hline & 3 & $3.83 \mathrm{E}-06$ & 4.00 & $2.10 \mathrm{E}-08$ & 4.99 & $1.64 \mathrm{E}-04$ & 3.00 & $8.76 \mathrm{E}-07$ & 4.02 \\
\hline & 4 & $2.39 \mathrm{E}-07$ & 4.00 & $6.71 \mathrm{E}-10$ & 4.96 & $2.05 \mathrm{E}-05$ & 3.00 & $5.46 \mathrm{E}-08$ & 4.00 \\
\hline \multicolumn{10}{|c|}{$\tau=1 / h$} \\
\hline \multirow{4}{*}{1} & 1 & $1.33 \mathrm{E}-01$ & - & $8.18 \mathrm{E}-03$ & - & $7.20 \mathrm{E}-01$ & - & $6.29 \mathrm{E}-02$ & - \\
\hline & 2 & $5.12 \mathrm{E}-02$ & 1.37 & $1.93 \mathrm{E}-03$ & 2.08 & $3.68 \mathrm{E}-01$ & 0.97 & $1.64 \mathrm{E}-02$ & 1.94 \\
\hline & 3 & $2.33 \mathrm{E}-02$ & 1.14 & $4.76 \mathrm{E}-04$ & 2.02 & $1.85 \mathrm{E}-01$ & 0.99 & $4.13 \mathrm{E}-03$ & 1.99 \\
\hline & 4 & $1.13 \mathrm{E}-02$ & 1.04 & $1.19 \mathrm{E}-04$ & 2.00 & $9.28 \mathrm{E}-02$ & 1.00 & $1.03 \mathrm{E}-03$ & 2.00 \\
\hline \multirow{4}{*}{2} & 1 & $1.50 \mathrm{E}-02$ & - & $3.59 \mathrm{E}-04$ & - & $9.89 \mathrm{E}-02$ & - & $3.40 \mathrm{E}-03$ & \\
\hline & 2 & $2.95 \mathrm{E}-03$ & 2.35 & $2.99 \mathrm{E}-05$ & 3.58 & $2.66 \mathrm{E}-02$ & 1.90 & $4.74 \mathrm{E}-04$ & 2.84 \\
\hline & 3 & $6.75 \mathrm{E}-04$ & 2.13 & $3.07 \mathrm{E}-06$ & 3.28 & $6.78 \mathrm{E}-03$ & 1.97 & $6.12 \mathrm{E}-05$ & 2.95 \\
\hline & 4 & $1.65 \mathrm{E}-04$ & 2.04 & $3.60 \mathrm{E}-07$ & 3.09 & $1.70 \mathrm{E}-03$ & 1.99 & 7.72E-06 & 2.99 \\
\hline \multirow{4}{*}{3} & 1 & $1.32 \mathrm{E}-03$ & - & $2.23 \mathrm{E}-05$ & - & $1.06 \mathrm{E}-02$ & - & $2.33 \mathrm{E}-0$ & - \\
\hline & 2 & $1.31 \mathrm{E}-04$ & 3.34 & $8.25 \mathrm{E}-07$ & 4.76 & $1.32 \mathrm{E}-03$ & 3.00 & $1.41 \mathrm{E}-0$ & 4.05 \\
\hline & 3 & $1.51 \mathrm{E}-05$ & 3.12 & $3.75 \mathrm{E}-08$ & 4.46 & $1.64 \mathrm{E}-04$ & 3.00 & $8.72 \mathrm{E}-0$ & 4.02 \\
\hline & 4 & $1.84 \mathrm{E}-06$ & 3.03 & $2.07 \mathrm{E}-09$ & 4.18 & $2.05 \mathrm{E}-05$ & 3.00 & $5.43 \mathrm{E}-0$ & 4.00 \\
\hline
\end{tabular}

The history of convergence of the HDG and the EDG methods is displayed in Table 4.1 and plotted in Figs. 4.2 and 4.3. In Table 4.1, we see that, for $\tau=h:=2^{-\ell}$ and $\tau=1$, the orders of convergence for the HDG method are $k+1$ and $k+2$ for the flux and the scalar variable, respectively, whereas those of the corresponding EDG method are $k$ and $k+1$ only, respectively. We also see that when we take $\tau=1 / h$, the orders of convergence of both HDG and EDG are $k$ and $k+1$ only, respectively. These results are in full agreement with our theoretical results.

In Figs. 4.2 and 4.3, we plot the results obtained in Table 4.1 in terms of the computational complexity of the methods in order to have a better idea of the relative efficiency of the HDG and EDG methods. We define the computational complexity 
of the methods as the number of operations for convergence of the conjugate gradient (CG) method without preconditioner. We compute this number by multiplying the number of nonzero entries of the stiffness matrix by the number of iterations for convergence of the CG method. The initial guess for the CG method is zero and its iterations are stopped whenever the ratio of the $\ell^{2}-$ norm of the residual of the current iterate to that of the initial one is smaller than $10^{-12}$. In Table 4.2, we display the condition numbers of the stiffness matrices as well as the numbers of iterations needed for the convergence of the CG method for the HDG and the corresponding EDG method for $\tau=1$. We do not display the results for $\tau=h$ and $\tau=h^{-1}$ as they are very similar. Note that the condition number of the HDG method is bigger than that of the EDG method, as expected. Note also that the square root of the ratio of the condition numbers is very close to the ratio of the numbers of iterations for convergence. This shows that our implementation of the CG method is reliable since the method converges in a number of iterations agreeing with the theory.

TABLE 4.2

Condition numbers and number of iterations for convergence of the CG method for $\tau=1$.

\begin{tabular}{|c|c|c|c|c|c|c|c|}
\hline & \multicolumn{4}{|c|}{ Condition numbers $\kappa$} & \multicolumn{3}{|c|}{ Iterations for convergence $N$} \\
\hline$k$ & $\ell$ & $\kappa_{\mathrm{HDG}}$ & $\kappa_{\mathrm{EDG}}$ & $\sqrt{\kappa_{\mathrm{HDG}} / \kappa_{\mathrm{HDG}}}$ & $N_{\mathrm{HDG}}$ & $N_{\mathrm{EDG}}$ & $N_{\mathrm{HDG}} / N_{\mathrm{HDG}}$ \\
\hline \multirow{4}{*}{1} & 1 & $6.32 \mathrm{E}+01$ & $5.80 \mathrm{E}+00$ & 3.30 & 46 & 9 & 5.11 \\
\hline & 2 & $2.48 \mathrm{E}+02$ & $2.53 \mathrm{E}+01$ & 3.13 & 91 & 27 & 3.37 \\
\hline & 3 & $9.85 \mathrm{E}+03$ & $1.03 \mathrm{E}+02$ & 3.09 & 180 & 56 & 3.21 \\
\hline & 4 & $3.93 \mathrm{E}+04$ & $4.14 \mathrm{E}+02$ & 3.08 & 358 & 118 & 3.03 \\
\hline \multirow{4}{*}{2} & 1 & $1.11 \mathrm{E}+02$ & $2.74 \mathrm{E}+01$ & 2.00 & 59 & 33 & 1.79 \\
\hline & 2 & $4.26 \mathrm{E}+03$ & $1.09 \mathrm{E}+02$ & 1.97 & 117 & 60 & 1.95 \\
\hline & 3 & $1.70 \mathrm{E}+04$ & $4.39 \mathrm{E}+02$ & 1.96 & 223 & 122 & 1.82 \\
\hline & 4 & $6.75 \mathrm{E}+04$ & $1.76 \mathrm{E}+03$ & 1.96 & 464 & 249 & 1.86 \\
\hline \multirow{4}{*}{3} & 1 & $1.75 \mathrm{E}+02$ & $6.84 \mathrm{E}+01$ & 1.60 & 73 & 50 & 1.46 \\
\hline & 2 & $6.82 \mathrm{E}+03$ & $2.76 \mathrm{E}+02$ & 1.57 & 142 & 98 & 1.45 \\
\hline & 3 & $2.71 \mathrm{E}+04$ & $1.11 \mathrm{E}+03$ & 1.56 & 280 & 199 & 1.41 \\
\hline & 4 & $1.08 \mathrm{E}+05$ & $4.43 \mathrm{E}+03$ & 1.56 & 567 & 400 & 1.42 \\
\hline
\end{tabular}

In Fig. 4.2, we compare methods using the same polynomial degree for the unknown $\widehat{u}_{h}$; since the dependence on $\tau$ of our measure of computational complexity and of the approximations given by the EDG methods seems to be small, we have only plotted the results for $\tau=1$. We see that the EDG is less efficient than any of the HDG methods (except for $k=1$ for the approximation of $u$ ), and that the HDG method seems to be more efficient for values of $\tau$ that are not too big. We also see that the approximations provided by the HDG methods with $k \geq 2$ do not seem to be very sensitive as $\tau$ varies from 1 to $h$. In Fig. 4.3, we compare the efficiency of the HDG and EDG methods for $\tau=1$. We see that the approximation provided by the HDG method using polynomials of degree $k$ is comparable, although slightly less efficient, than the approximation of the EDG method using polynomials of degree $k+1$.

5. Concluding remarks. In this paper, we have shown that although HDG methods are computationally slower than the corresponding EDG methods, they can provide approximations converging with an additional order of convergence; in fact, they are more efficient.

We would like to end this paper with a brief speculation about why does this happen. In [10], it was pointed out that the reason HDG methods using polynomials of degree $k$ in both variables can provide approximations of order $k+2$ and $k+1$ for 

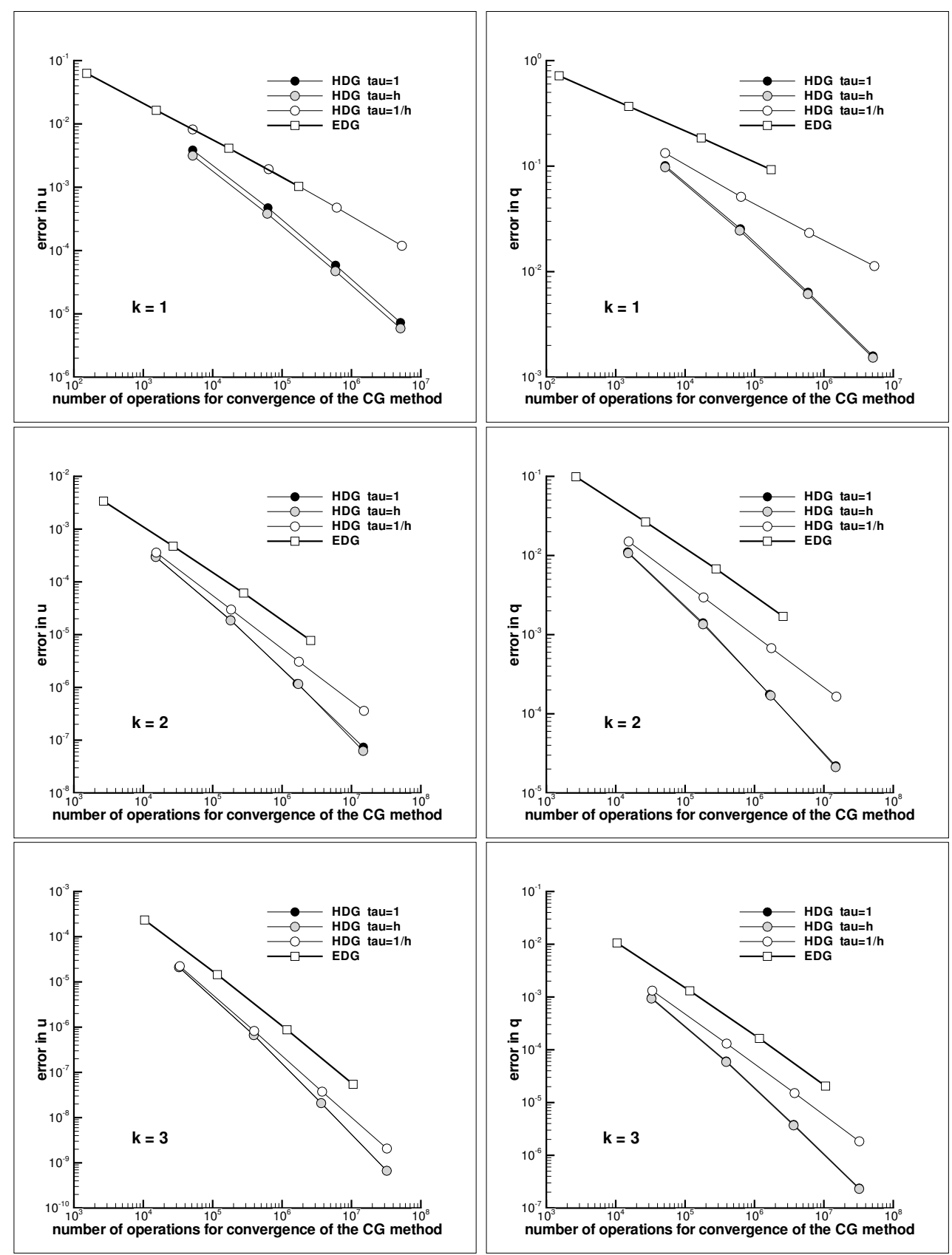

FIG. 4.2. History of convergence of the HDG methods (for different values of $\tau$ ) and the corresponding EDG method (for $\tau=1$ ) for the same fixed polynomial degree of the numerical trace $\widehat{u}_{h}$.

the potential and the flux, respectively, is due to the fact that the jump in the normal component of the approximate flux is small enough. This result, however, assumes the single-valuedness of the normal component of the numerical trace $\widehat{\boldsymbol{q}}_{h}$. The numerical trace of the EDG methods is, however, not single valued, and this seems to induce 

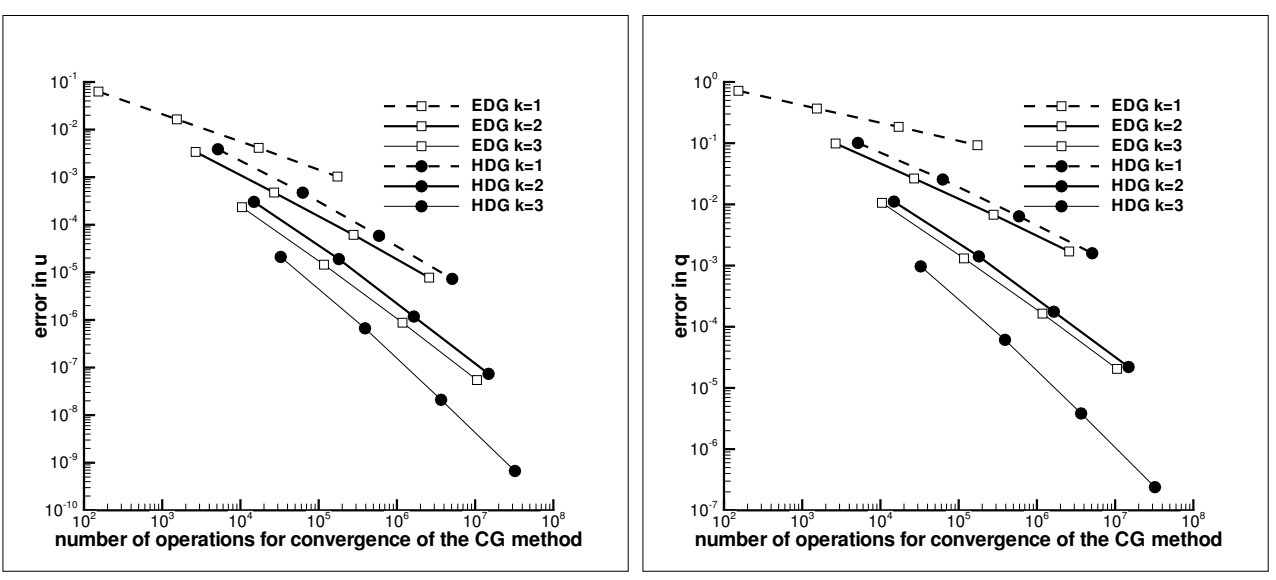

FIG. 4.3. History of convergence of the HDG and the corresponding EDG method when $\tau=1$.

the above-mentioned loss of accuracy.

For this reason, we surmise that EDG methods associated to the hybridized version of the Raviart-Thomas or the Brezzi-Douglas-Marini mixed methods might behave in the same way.

Acknowledgements. The authors would like to thank Sukru Güzey for bringing to their attention that his numerical experiments suggested that the numerical trace $\widehat{u}_{h}$ of the EDG method for $k=1$ coincides with the restriction to $\mathscr{E}_{h}$ of the approximation provided by the continuous Galerkin method.

Appendix: On the relation of the EDG and the continuous Galerkin methods. Here we would like to establish a simple but interesting result about the relation between the EDG and the continuous Galerkin methods. It relates the numerical flux $\widehat{u}_{h}^{\mathrm{EDG}}$ of the EDG method and the approximation $u_{h}^{\mathrm{G}}$ provided by the continuous Galerkin method.

Proposition 5.1. We have that $\widehat{u}_{h}^{\mathrm{EDG}}=u_{h}^{\mathrm{G}}$ on $\mathscr{E}_{h}$ for all data $g$, $\mathrm{q}_{\mathrm{N}}$ and $f$ if and only if $k=1$.

Proof. Let us begin by noting that, by definition of the continuous Galerkin method, we have that

$$
\left(\nabla u_{h}^{\mathrm{G}}, \nabla w\right)_{\Omega_{h}}=(f, w)_{\Omega_{h}},
$$

for all $w \in W_{h}^{0}$, where $W_{h}^{0}$ is the space of functions of $W_{h}$ which are continuous on $\Omega$ and equal to zero on $\partial \Omega_{D}$. By the second and third equations defining the EDG method, (2.1b) and (2.1c), we have that

$$
\left(\nabla u_{h}^{\mathrm{G}}, \nabla w\right)_{\Omega_{h}}=-\left(\boldsymbol{q}_{h}^{\mathrm{EDG}}, \nabla w\right)_{\Omega_{h}} .
$$

Now, denote by $\widehat{u}_{h}^{\mathrm{EDG}}$ any function in $W_{h}$ that coincides with $\widehat{u}_{h}^{\mathrm{EDG}}$ on $\mathscr{E}_{h}$. Then, we can write

$$
\left(\nabla\left(u_{h}^{\mathrm{G}}-\widehat{u}_{h}^{\mathrm{EDG}}\right), \nabla w\right)_{\Omega_{h}}=-\left(\boldsymbol{q}_{h}^{\mathrm{EDG}}+\nabla \widehat{u}_{h}^{\mathrm{EDG}}, \nabla w\right)_{\Omega_{h}} .
$$

We claim that, for $k=1$, the right-hand side of the above identity is equal to zero. This implies that $u_{h}^{\mathrm{G}}=\widehat{u}_{h}^{\mathrm{EDG}}$ on $\Omega$ and in particular on $\mathscr{E}_{h}$. 
Let us prove the claim. Note that by the first equation defining the EDG method, (2.1a), we have that

$$
\left(\boldsymbol{q}_{h}^{\mathrm{EDG}}+\nabla \widehat{u}_{h}^{\mathrm{EDG}}, \boldsymbol{v}\right)_{\Omega_{h}}=\left(u_{h}^{\mathrm{EDG}}-\widehat{u}_{h}^{\mathrm{EDG}}, \nabla \cdot \boldsymbol{v}\right)_{\Omega_{h}},
$$

for all $\boldsymbol{v} \in \boldsymbol{V}_{h}$. Hence, taking $\boldsymbol{v}=\nabla w$ where $w \in W_{h}^{0}$, we get

$$
\left(\boldsymbol{q}_{h}^{\mathrm{EDG}}+\nabla \widehat{u}_{h}^{\mathrm{EDG}}, \nabla w\right)_{\Omega_{h}}=\left(u_{h}^{\mathrm{EDG}}-\widehat{u}_{h}^{\mathrm{EDG}}, \Delta w\right)_{\Omega_{h}}=0,
$$

if $k=1$. This proves the claim.

To show that we need $k=1$ to have $\widehat{u}_{h}^{\mathrm{EDG}}=u_{h}^{\mathrm{G}}$ on $\mathscr{E}_{h}$ for any data, we construct a counter-example. Thus, we take $\Omega$ to be a polygon that can be meshed with only two triangles, $T_{1}$ and $T_{2}$. We take homogeneous boundary conditions, $\partial \Omega_{N}=\emptyset$, and $f=0$ on $T_{1}$ and nonzero on $T_{2}$. We consider the EDG method associated to the single-face hybridizable ( $\mathrm{SFH}$ ) method with $k=2$ studied in [7].

In this case, the equation (5.1) becomes

$$
\left(\nabla\left(u_{h}^{\mathrm{G}}-\widehat{u}_{h}^{\mathrm{EDG}}\right), \nabla w\right)_{\Omega_{h}}=-\sum_{i=1,2}\left(\boldsymbol{q}_{h}^{\mathrm{EDG}}+\nabla \widehat{u}_{h}^{\mathrm{EDG}}, \nabla w\right)_{T_{i}},
$$

and, by the equation (5.2),

$$
\begin{aligned}
\left(\nabla\left(u_{h}^{\mathrm{G}}-\widehat{u}_{h}^{\mathrm{EDG}}\right), \nabla w\right)_{\Omega_{h}}= & -\left(\boldsymbol{q}_{h}^{\mathrm{EDG}}+\nabla \widehat{u}_{h}^{\mathrm{EDG}}, \nabla w\right)_{T_{1}} \\
& +\left(u_{h}^{\mathrm{EDG}}-\widehat{u}_{h}^{\mathrm{EDG}}, \Delta w\right)_{T_{2}},
\end{aligned}
$$

for all $w \in W_{h}^{0}$. Note that the dimension of the space $W_{h}^{0}$ is one. Note also that if we take $T_{2}$ to be a righ-angled isosceles triangle whos two equal sides are boundary edges, then $\Delta w=0$ on $T_{2}$. This implies that we have

$$
\left(\nabla\left(u_{h}^{\mathrm{G}}-\widehat{u}_{h}^{\mathrm{EDG}}\right), \nabla w\right)_{\Omega_{h}}=-\left(\boldsymbol{q}_{h}^{\mathrm{EDG}}+\nabla \widehat{u}_{h}^{\mathrm{EDG}}, \nabla w\right)_{T_{1}} .
$$

Now, since $f=0$ on $T_{1}$ and since we are dealing with the SFH method, we have that $\nabla \cdot \boldsymbol{q}_{h}^{\mathrm{EDG}}=0$ on $T_{1}$. Since, by equation (5.2), we have that

$$
\left(\boldsymbol{q}_{h}^{\mathrm{EDG}}+\nabla \widehat{u}_{h}^{\mathrm{EDG}}, \boldsymbol{v}\right)_{\Omega_{h}}=0,
$$

for all $\boldsymbol{v} \in \boldsymbol{V}_{h}$ whose divergence is zero on each element, we obtain that

$$
\boldsymbol{q}_{h}^{\mathrm{EDG}}=-\boldsymbol{P} \nabla \widehat{u}_{h}^{\mathrm{EDG}},
$$

where $\boldsymbol{P}$ is the $L^{2}$-projection into the space of functions $\boldsymbol{V}_{h}$ whose divergence is zero on each element. We thus have that

$$
\begin{aligned}
\left(\nabla\left(u_{h}^{\mathrm{G}}-\widehat{u}_{h}^{\mathrm{EDG}}\right), \nabla w\right)_{\Omega_{h}} & =\left(\boldsymbol{P} \nabla \widehat{u}_{h}^{\mathrm{EDG}}-\nabla \widehat{u}_{h}^{\mathrm{EDG}}, \nabla w\right)_{T_{1}}, \\
& =(\boldsymbol{P} \nabla w-\nabla w, \nabla w-\boldsymbol{P} \nabla w)_{T_{1}}\left(\widehat{u}_{h}^{\mathrm{EDG}}\right)(\boldsymbol{m})
\end{aligned}
$$

where $\boldsymbol{m}$ is the midpoint of the interior edge. This implies that

$$
\left(u_{h}^{\mathrm{G}}-\widehat{u}_{h}^{\mathrm{EDG}}\right)(\boldsymbol{m})=-\frac{\|\boldsymbol{P} \nabla w-\nabla w\|_{L^{2}\left(T_{1}\right)}^{2}}{\|\nabla w\|_{L^{2}\left(\Omega_{h}\right)}^{2}} \widehat{u}_{h}^{\mathrm{EDG}}(\boldsymbol{m}) .
$$

Clearly $\widehat{u}_{h}^{\mathrm{EDG}}(\boldsymbol{m})$ is not zero if we take $f>0$ on $T_{2}$. Moreover, since $\boldsymbol{P} \nabla w=\nabla w$ if and only if $\Delta w=0$, if we take $T_{1}$ such that the angle facing its interior edge be different from $\pi / 2$, we would have that $\left(u_{h}^{\mathrm{G}}-\widehat{u}_{h}^{\mathrm{EDG}}\right)(\boldsymbol{m}) \neq 0$. This completes the proof of Proposition 5.1. 


\section{REFERENCES}

[1] D. N. Arnold, F. Brezzi, B. Cockburn, and L. D. Marini, Unified analysis of discontinuous Galerkin methods for elliptic problems, SIAM J. Numer. Anal. 39 (2002), 1749-1779.

[2] F. Brezzi, J. Douglas, Jr., and L. D. Marini, Two families of mixed finite elements for second order elliptic problems, Numer. Math. 47 (1985), 217-235.

[3] A. Buffa, T. J. R. Hughes, and G. Sangalli, Analysis of a multiscale discontinuous Galerkin method for convection-diffusion problems, SIAM J. Numer. Anal. 44 (2006), 1420-1440 (electronic).

[4] P. Castillo, B. Cockburn, I. Perugia, and D. Schötzau, An a priori error analysis of the local discontinuous Galerkin method for elliptic problems, SIAM J. Numer. Anal. 38 (2000), $1676-1706$.

[5] B. Cockburn and B. Dong, An analysis of the minimal dissipation local discontinuous Galerkin method for convection-diffusion problems, J. Sci. Comput. 32 (2007), 233-262.

[6] B. Cockburn, B. Dong, and J. Guzmán, Optimal convergence of the original DG method for the transport-reaction equation on special meshes, SIAM J. Numer. Anal. 46 (2008), 12501265 .

[7] — A superconvergent LDG-hybridizable Galerkin method for second-order elliptic problems, Math. Comp. 77 (2008), 1887-1916.

[8] B. Cockburn and J. Gopalakrishnan, A characterization of hybridized mixed methods for second order elliptic problems, SIAM J. Numer. Anal. 42 (2004), 283-301.

[9] B. Cockburn, J. Gopalakrishnan, and R. Lazarov, Unified hybridization of discontinuous Galerkin, mixed and continuous Galerkin methods for second order elliptic problems, SIAM J. Numer. Anal., to appear.

[10] B. Cockburn, J. Guzmán, and H. Wang, Superconvergent discontinuous Galerkin methods for second-order elliptic problems, Math. Comp. 78 (2009), 1-24.

[11] S. Güzey, B. Cockburn, and H.K. Stolarski, The embedded discontinuous Galerkin methods: Application to linear shells problems, Internat. J. Numer. Methods Engrg. 70 (2007), 757790 .

[12] S. Güzey, H. K. Stolarski, B. Cockburn, and K. K. Tamma, Design and development of discontinuous Galerkin methods for shells, Comput. Methods Appl. Mech. Engng. 195 (2006), 3183-3550, C. Dawson, Ed.

[13] J. T. R. Hughes, G. Scovazzi, P. B. Bochev, and A. Buffa, A multiscale discontinuous Galerkin method with the computational structure of a continuous Galerkin method, Comput. Methods Appl. Mech. Engng. 195 (2006), 2761-2787.

[14] P. A. Raviart and J. M. Thomas, A mixed finite element method for second order elliptic problems, Mathematical Aspects of Finite Element Method (I. Galligani and E. Magenes, eds.), Lecture Notes in Math. 606, Springer-Verlag, New York, 1977, pp. 292-315.

[15] S.J. Sherwin, R.M. Kirby, J. Peiró, R.L. Taylor, and O.C. Zienkiewicz, On $2 D$ elliptic discontinuous Galerkin methods, Internat. J. Numer. Methods Engrg. 65 (2006), no. 5, 752-784. 